\title{
UNA PERDUTA ISCRIZIONE A PAOLO PARUTA SULLE LAPIDI DEL CORTILE DEL BROLETTO DI BRESCIA ${ }^{*}$
}

\author{
MARCO GIANI (*) \\ Nota presentata dal s.c. Francesco Bruni \\ (Adunanza del 1 marzo 2018)
}

SunTO. - Grazie ad un passo delle Coryat's Crudities (1611), in cui il viaggiatore inglese descrive la sua visita a Brescia (svoltasi nell'agosto del 1608), è ora possibile ricostruire una lapide commemorativa che si trovava un tempo sul muro interno del Broletto locale. L'iscrizione, che si trovava sulla lapide marmorea (datata 1591), fu cancellata quasi un secolo dopo (1692) dalle autorità della Repubblica di Venezia, preoccupate per la crescente auto-glorificazione dei magistrati patrizi. Per fortuna, Coryate trascrisse l'iscrizione, dedicata a Paolo Paruta (1540-1598), uomo politico e storiografo della Repubblica di Venezia, capitano di Brescia dal 1590 al 1592, quando fu nominato Ambasciatore a Roma. Partendo dalla riscoperta dell'iscrizione perduta, il presente lavoro cercherà di comprendere come Paolo Paruta, un acuto scrittore di politica ma anche un abile uomo di stato, svolse il proprio incarico a Brescia, allora per importanza la seconda città della Terraferma veneziana.

$* * *$

ABSTRACT. - Thanks to a passage of Coryat's Crudities (1611), where the English traveller describes his visit to Brescia (August 1608), now is possible to reconstruct a lost commemorative plaque, once placed upon the internal wall of the local Broletto (City Hall). The inscription upon that marble plaque, dated 1591, was erased almost one hundred years later (1692) by the authorities of Venetian Republic, worried for the growing self-glorification of patrician magistrates. Luckily Coryat transcribed the inscription, dedicated to Paolo Paruta (1540-1598), statesman and historiographer of the Venetian Republic, capitano of Brescia from 1590 to 1592, when he was appointed

(*) Ricercatore Indipendente, Italy. E-mail: gianimarco@gmail.com

\# Le trascrizioni sono state condotte secondo i criteri esposti nell'Appendice di GIANI 2017. 
as Ambassador to Rome. Starting from the discovery of this lost inscription, this essay tries to investigate how Paolo Paruta, a refined political writer but also an able statesman, took his 2 year office in Brescia, at that time the second city of Venetian Terraferma.

\section{LE LAPIDI «SCANSELLATE» DEL BROLETTO BRESCIANO}

Le numerose lapidi che oggi accolgono il visitatore nel cortile interno del Broletto di Brescia risultano illeggibili perché abrase nel 1692. Quell'anno, infatti, come narrato da un cronista cittadino in data 4 marzo,

vengono scansellate ed abbollite quelle pietre, sopra de' quali in Broletto si vedevano Eloggi et inscritioni in lode de' Rappresentanti passati, ove si ponno vedere anche quei marmi ove erano impresse tutti scancellati ${ }^{1}$

La cancellazione del contenuto delle lapidi fu ordinata il 15 dicembre del 1691 dal Senato della Repubblica di Venezia per porre fine ad un'usanza che aveva preso piede soprattutto lungo quel XVII secolo che si stava per chiudere. Le lodi ai magistrati veneziani delle città di Terraferma, infatti, erano state immortalate nelle chiese o in luoghi pubblici su bassorilievi in pietra oppure, nella forma delle armi familiari, in sculture. ${ }^{2}$ Se già localmente si era provato a rimediare all' $a$ buso (come accaduto ad esempio a Rovigo, dove, negli anni Quaranta, le armi erano state rimosse dagli edifici pubblici), ${ }^{3}$ a fine secolo lo scandaloso disordine doveva aver evidentemente ormai travalicato ogni sorta di limite (con intolerabile eccesso, come il dice il testo stesso della provisione 'deliberazione' del Senato). ${ }^{4}$ La Repubblica, che avrebbe pur tollerato la lode rivolta al magistrato più che all'uomo privato, ${ }^{5}$ decise di

1 GUERRINI 1922:1692. Per la vicenda della cancellatura delle lapidi a Brescia, vd. VOLTA 1987:27, ZANe 1998:8.

2 RAINES 2006:212-225.

3 RAINES 2006:218.

4 In NetTO 1991-1992:29-31 è pubblicato per intero il testo della provisione, seguito dalla ducale (di qualche giorno posteriore) del doge Francesco Morosini al Podestà di Treviso Andrea Stazio.

5 RAINES 2006:219-220. 
combattere una pratica che appariva tanto contraria sia all'egualità interna al patriziato, ${ }^{6}$ sia all'immagine pubblica di un governo impersonale e perciò latore ai propri sudditi di una giustizia imparziale. Come dice la provisione stessa,

onde più non sussista apparenza alcuna di queste memorie e tutto sia ridotto a semplice nudo ornamento de' Palazzi, consistendo senz'altra vana ostentatione il vero monumento nella buona impressione che lascia nel cuore de' sudditi la retta giustizia de' Rappresentanti

In realtà, l'ordine del Senato venne poi applicato in maniera assai diversa nei vari centri del dominio della Serenissima, ${ }^{7}$ ma lo fu in maniera particolarmente zelante all'interno del grande cortile del Broletto di Brescia, sede cittadina dei due rettori veneti - anche se va notato che «questo scempio ha colpito in misura maggiore il lato interno dell'ala orientale, proprio perché qui le iscrizioni erano più numerose. I Giacobini fecero il resto». ${ }^{8}$

La bontà (se così si può dire) del lavoro è testimoniata dal fatto che attualmente tutte le lapidi del cortile risultano completamente illeggibili. ${ }^{9}$ Per questo il tentativo di Valentino Volta di ricostruirne il

6 Giustamente, in Giové MarChIOLI 2010:14, si afferma che la provisione del 1692, più che al modello classico della damnatio memoriae di un singolo personaggio, sia da ricondurre a quella deprecazione del culto della personalità che in epoca medievale si era espresso ad es. nella legislazione antimagnatizia. Per un esempio di tale tensione nel pensiero politico dello stesso Paolo Paruta vd. GIANI 2014.

7 Rizzi ha dimostrato come, a differenza di quanto sostenuto in precedenza da Passamani, quella di Brescia non fu un'eccezione: semplicemente, «tali incongrui moralistici provvedimenti furono adottati con un andamento a "macchia di leopardo", secondo cioè lo zelo dei singoli rettori preposti a cui si aggiungono le complicazioni territoriali e giuridiche delle loro competenze» (RIzZI 1994-1995:629-630). Nello stesso studio è contenuta una rassegna dell'applicazione della provisione del dicembre 1691 nei vari centri di Terraferma, che può essere arricchita con il ricorso a successivi studi riguardanti le singole città, come ad es. Belluno (GIOvé MARCHIOLI 2010:142), Feltre (Corazzol 1997:84-85), Padova (Benucci 2001:245-247, ma per la Padova universitaria BENUCCI 2007:304).

8 PiOvanelli 1975:19. Sull'applicazione della provisione a Brescia vd. anche Giové MARCHIOLI 2010:141-142. Sulla «leontoclastìa giacobina del 1797» (che appunto colpì i leoni di San Marco come simboli dell'intera Repubblica, non dei singoli rettori) vd. invece RIZZI 1994-1995, GIOvÉ MaRCHIOLI 2010:145.

9 La provisione del 1691 così recitava, riguardo le lapidi, dopo aver parlato 
contenuto si è dovuto basare su testimonianze indirette: per fortuna, qualcuno, prima del 1692, si era preso la briga di trascrivere per $\mathrm{i}$ posteri quanto poteva allora leggere senza problemi entrando nel cortile.

Alla breve rassegna di Volta, basata su «poche tracce nei documenti dell'Archivio Storico Civico», ${ }^{10}$ possiamo ora aggiungere un'altra, fornita da un testo letterario inglese di inizio Seicento. Il magistrato veneto lodato è Paolo Paruta (1540-1598), più volte ambasciatore della Repubblica, nonché Storiografo Pubblico della stessa. ${ }^{11}$

\section{Paolo Paruta Capitano di Brescia (1590-1592)}

\subsection{La cronologia dell'incarico}

Como noto, la carica di capitan(i)o di Brescia durava normalmente 16 mesi. ${ }^{12}$ L'elezione di Paolo Paruta risale all'agosto del 1590: ${ }^{13}$ andava quindi a collaborare col podestà Tommaso di Almorò Contarini, eletto a fine aprile. ${ }^{14}$ Avendo potuto di fatto trasferirsi nella città lombarda solo a fine dell'anno ${ }^{15}$ (a causa di vari problemi legati

delle statue: «siano in oltre cancellate et abolite tutte l'inscrittioni che per ogni altra figura, ritratto o arma rimanessero». Nella ducale ad Andrea Stazio, molto più sinteticamente: «l'abolitione di tutte l'inscrittioni».

10 VOLTA 1987:27.

11 Per la sua biografia, vd. BENZONI 2014, da integrare con GIANI 2012:7-23.

12 ManNo 1987:263.

13 Poco prima della nomina Paruta era ancora a Venezia, come dimostrato dal fatto che il 30 luglio 1590 è segnalato come compare (cioè 'padrino di battesimo') di una figlia del pittore Francesco Bassano, presso la parrocchia di San Canciano: vd. il documento pubblicato in SAPIENZA 2007/2008:13.

14 Dispacci, III, 143. Per quanto riguarda il Morosini, è lui stesso, in un dispaccio datato 13/11/1590, a ricordare come sia giunto a Brescia il 16 settembre (Capi di X, 17, c. 1r). I due nuovi rettori andavano a sostituire la coppia formata dal podestà Lorenzo Priuli e dal capitano Niccolò Gussoni per il biennio 1589/1590: vd. BonELLI 1924.

15 Ragionevolmente, questo è il motivo per cui alcune fonti (ad es. la lista dei Rettori di Brescia presente in BONELLI 1924), dichiarano che Paolo Paruta fu capitano di Brescia dal 1591 al 1592. In BENZONI 2014 la carica bresciana è data «dall'inizio del 1591 quanto meno sino al giugno del 1592». 
al trasporto delle sue suppellettili), ${ }^{16}$ Paruta manterrà la carica fino alla primavera del 1592, quando verrà eletto Ambasciatore ordinario a Roma (1592-1595). ${ }^{17}$ Eccettuato un ritorno affrettato in Laguna nello scorcio finale della primavera del 1592, i due incarichi furono quindi praticamente consecutivi, come denunciato dall'ambasciatore stesso in un dispaccio del settembre $1595 . .^{18}$

16 In una lettera datata 23 novembre 1590 (pubblicata in BIADEGO 1885: 1011), Paruta racconta al conte veronese Federico Serego di come stia scrivendo ancora da Venezia a causa della tardità 'ritardo' del burchio che doveva occuparsi del trasloco dei suoi averi nella città lombarda: conta comunque di poter partire il 3 o il 4 dicembre.

17 Eletto per l'incarico romano il 30 aprile 1592, la presenza del Paruta a Brescia è documentata ancora per un mese, cioè fino al 30 maggio (BENZONI 2014). Un documento del Senato veneziano datato 31 maggio 1592 spiega i problemi venutisi a creare in quei giorni con la morte del precedente ambasciatore a Roma, Giovanni Moro. In quel momento, infatti, nella Città Eterna si trovava già Leonardo Donà, Ambasciatore straordinario (assieme a Zaccaria Contarini, Alberto Badoer e Marin Grimani) per complimentarsi dell'elezione di Clemente VIII: il Senato ordinò a Donà di trattenersi temporaneamente a Roma, «non essendo il Paruta, eletto suo successore, in termine de andarvi così presto» (cit. in SENECA 1959:206-207). Si è conservata la parte del Senato di fine agosto 1592 che stanzia dei fondi per il nuovo incarico di Paruta: «Dovendosi spedire per la sua Ambasceria di Roma il dilettissimo nobile nostro Polo Paruta, I L'anderà parte che delli danari a questo deputati siano dati a lui [...] Ambasciator Paruta, o a suoi legitimi commessi ducati ottocento d'oro in oro a conto di sue spese per mesi quattro in ragion di ducati duecento d'oro in oro al mese, senza obligo di renderne conto alcuno, et ducati mille d'oro per donativo secondo la forma della parte del Senato di 2 Giugno 1561. I Per cavalcature, coperte et forcieri ducati trecento, et per spese estraordinarie ducati trecento, delle quali sia tenuto render conto [...]. I Et acciò possa comparere nell'istesso habito che compariranno gl'Ambasciatori nostri estraordinarij destinati per la creatione del Sommo Pontefice ducati cento da farsi anch'esso un manto et ducati quaranta per staffieri, come si osserva in casi simili. I $\mathrm{Al}$ secretario suo in dono ducati cento, et al cogitor cinquanta per mettersi all'ordine. | Per dui corriere che lo accompagneranno ducati vinti» (Senato Terra, filza 123, 29/08/1592). Già in data $1^{\circ}$ luglio 1592, comunque, il successore di Paruta si firma come «Benetto Moro Capitano» in una missiva circa «questo edificio de la Polvere»: vd. dispaccio conservato in Senato Terra, filza 121.

18 «Faccia il Signor Dio che, dopo lo spazio di tempo quasi di cinque anni, ne' quali, posso dire che quasi continuamente, rispetto per il breve tempo che io mi fermai in quella città tra il reggimento di Brescia e questa ambasceria, sono stato fuori occupato in publici carichi, possa ritornare a Vostra Serenità con salute, e in stato di poter presso di lei medesima continuare il mio umile e riverente servizio» (cit. da Dispacci, t. III, p. 300). 


\subsection{L'importanza dell'incarico}

La nomina a capitano (praefectus, ${ }^{19}$ nella forma latinizzata di cui avremo modo di parlare successivamente) fu sicuramente un'importante tappa del cursus honorum di Paruta, che già aveva avuto occasione (visti i suoi numerosi incarichi come Savio di Terraferma nel corso degli anni Ottanta ${ }^{20}$ di conoscere i domini di Terraferma, e che, dopo il biennio bresciano avrebbe fatto il definitivo salto di qualità come uomo politico della Serenissima proprio con l'ambasceria romana.

Dall'alto dei suoi 35.500 abitanti $^{21}$ Brescia, all'epoca, era seconda solo a Padova fra i centri di Terraferma: una sede quindi prestigiosa ${ }^{22}$ ma anche molto impegnativa e per questo abhorrita dai chi veniva mandato a reggere la città. ${ }^{23}$ Affiancando il podestà Tommaso Morosini, Paruta avrebbe dovuto occuparsi, per almeno 16 mesi, soprattutto delle funzioni finanziarie e militari, lasciando al primo quelle civili e giudiziarie. ${ }^{24}$

\subsection{Profilo storico dell'incarico bresciano}

\subsubsection{Introduzione}

Per arrivare a commentare le lodi a Paruta come rettore di Brescia, sarà necessario capire cosa egli fece, nel corso di quei due anni. Tuttavia

19 A suo tempo già il Pompeati aveva corretto lo Zeno circa l'uso scorretto della forma volgare prefetto, anziché capitano: POMPEATI 1905:65.

20 BENZONI 2014, che riporta le date delle varie elezioni, ossia 27/11/1582, $11 / 06 / 1583,03 / 03 / 1584,31 / 12 / 1584,31 / 12 / 1586$ e 30/12/1589. Si è conservata una lettera di Gabriele Calzoni al Duca di Mantova, datata 6 luglio 1585, che in cui si racconta come la Repubblica di Venezia avesse inviato «il Lippomani et il Paruta Savi di Terra ferma a visitare il signor Pavolo Giordano [...]» (MANTOVA, Archivio di Stato, Archivio Gonzaga, b. 1515, fasc. I, c. 212r). Si tratta quindi di trattative per una condotta militare: nel prosieguo si dice che il Giordano voleva «ottenere il carico che aveva il già signor Sforza Pallavicino» (c. 212v).

21 AAVV 1978:154.

22 RAINES 2006:282.

23 Questi i sentimenti di Leonardo Donà, mandato come podestà a Brescia durante gli anni Ottanta: «Si trattava di un incarico pesante e certamente non desiderato, né, tanto meno, sollecitato, "abhorrito" da molti anzi, che relegava il Donà in uno dei settori più delicati dello Stato veneto» (SENECA 1959:118).

24 BALISTRERI 2015:77. 
purtroppo, ad oggi, non esiste ancora uno studio su Paolo Paruta capitano a Brescia che, analogamente a quanto fatto a suo tempo da Rinaldo Fulin con l'ambasceria romana, presenti le vicende storiche affrontate dal politico veneto grazie allo studio dei dispacci, che attualmente giacciono ancora inediti - e di fatto non ancora studiati - presso l'Archivio di Stato di Venezia. ${ }^{25}$ Tuttavia, unendo un primo (parziale) spoglio di questi documenti ad alcuni materiali già pubblicati, è possibile provare a tracciare un breve profilo dell'incarico bresciano, così da iniziare a comprendere quali problemi Paruta si trovò a gestire nella città lombarda, e come provò a risolverli. Si tratterà cioè di dare una concretezza storica a quella veneziana prudenza per nulla ideale bensì esercitata concretamente a Brescia per il quale il patrizio venne all'epoca lodato; ${ }^{26}$ così facendo, si ricostruirà un ulteriore ${ }^{27}$ tassello della biografia politica parutiana, con lo scopo ultimo di sottolineare come la riflessione politica degli anni Ottanta e Novanta di Paolo Paruta (poi rifluita nei Discorsi Politici, pubblicati postumi nel 1599) sia da considerare un tutt'uno con la concreta gestione dello stato territoriale veneziano di fine secolo. Il riapparire, in tale corpus amministrativo, dello stesso lessico politico "alto" dei venticinque testi del 1599 sarà la cartina al tornasole della bontà di tale ipotesi interpretativa.

\subsubsection{La produzione delle armi}

Per questioni di spazio, bisognerà in questa sede individuare solo

25 Tutti i documenti riguardanti la carica bresciana del Paruta sono stati inventariati in BENZONI 2014, a cui tuttavia bisogna aggiungere i seguenti due dispacci (forse sfuggiti al regesto perché firmati «i Rettori» o riportanti le firme prima del Morosini e poi del Paruta): nella filza 121, il dispaccio del 07/06/1591; nella filza 122, il dispaccio del 15/01/1592 (da intendere non more veneto:, come dimostrato per altro dal precedente documento, citato nel dispaccio, datato 14/12/1591). Bisogna infine notare che vi sono due dispacci (non uno solo) datati 19 novembre 1591.

26 Ai primi di giugno del 1592 il successore di Paruta nel capitanato di Brescia, Benedetto Moro, ricorda come «questa Città è stata sin hora governata da questo Clarissimo signor Podestà, et dal Clarissimo Paruta mio Precessore con quella prudenza che conviene a l'importanza di un tanto carico» (Capi di X, 53, 04/06/1592). Nella Nuova informatione sulla vita del padre, Giovanni Paruta dice brevemente che Paolo «fu mandato Capitano a Brescia, nel quale tempo quella città stete sempre in pace, avenendo ciò per la particolar prudentia del Capitano» (NuovaInfo, 1).

27 Per un primo esempio di questo tipo di approccio vd. il mio studio sul sostantivo prudenza e sugli incarichi annonari di Paruta a metà anni Novanta: GIANI 2013/2014. 
le questioni principali affrontate da Paruta come capitano di Brescia, tralasciandone altre (che tuttavia, per amor di completezza, verranno riassunte alla fine della rassegna).

Nel primo gruppo, la più importante appare essere senza dubbio quella della produzione delle armi nel Bresciano. Che l'argomento fosse stato particolarmente caro a Paruta lo si comprende bene da un dispaccio romano del maggio 1595, in cui egli stesso (ormai Ambasciatore ordinario presso Clemente VIII) ${ }^{28}$ ricorda - non richiesto - il proprio operato:

ancorché ciò sia fuori del carico che al presente io tengo, non è però oltre quell'obbligo universale e naturale, che debbo, di pensare e procurare sempre quello che possa tornare a servizio delle cose pubbliche; ma tanto più avendo, mentre mi trovai nel reggimento di Brescia, praticata lungamente e particolarmente questa materia

Segue difatti una dettagliata analisi dell'arte del fabbricare armi da dosso nel Bresciano, della sua crisi dopo il tempo dell'ultima peste (quella di metà anni Settanta) e infine dei possibili rimedi per far rifiorire tale un tempo fiorente attività. ${ }^{29}$

Se l'argomento è solo accennato nel carteggio romano, i dispacci permettono al contrario di farsi un'idea chiara ed ampia dell'operato parutiano. Prima di tutto, già nel luglio 1591 i Rettori (così spesso si firmano, al posto di farlo separatamente coi propri nomi e cognomi) introducono la materia delle ferrarezza $a^{30}$ che causa tanti danni a Brescia, non solo per il tramonto dell'attività manifatturiera (declinare tale arte), per i

28 Da segnalare come, durante il proprio incarico romano, Paruta avrà numerosissime occasioni di collaborazione con Giovanni Francesco Morosini, vescovo di Brescia proprio a partire da quel 1590 in cui anche l'uomo politico veneziano era arrivato a Brescia. Sull'episcopato del Morosini vd. MONTANARI 2007:335.

29 Dispacci, t. III, pp. 142-143. Si ricordi come, nel 1590, Paruta avesse svolto l'incarico di Provveditore sopra l'Artiglieria (vd. BEnzoni 2014). Per comprendere l'importanza economica della produzione e del commercio di armi e ferrarezze per la Brescia di quell'epoca, vd. BAUCE 2010:38-41. Il legame affettivo con Brescia può forse essere intravisto in un passaggio dei Discorsi Politici (pubblicati postumi, nel 1599, a Venezia) in cui Paruta, parlando della sconfitta di Agnadello, pone Brescia come primo ed unico esempio, in una lista di città di Terraferma: vd. DP II.III,9.

30 Il sostantivo ferrarezza può essere glossato come 'prodotti metallici' (così ad es. MAINONI 1997:15), oppure come 'arnesi di ferro di diverso genere' (così MAZZOLDI 1964:128). 
minori introiti fiscali (deterioramento de' suoi datij) e per il danno inferto al popolo, ma soprattutto per il venir meno di «quella cosa che è tanto in ogni stato necessaria, et della quale questo paese è stato particolarmente gratiato dalla natura, cioè delle arme da offesa et da difesa». ${ }^{31}$ Si ricordi come solo qualche anno prima, nel 1588, la Serenissima avesse istituito proprio a Brescia un fontego 'deposito' per «immagazzinare e custodire le canne prodotte nei tempi di magre richieste, in attesa di essere utilizzate»: fra i motivi del provvedimento pubblico, c'era quello di «evitare l'espatrio dei maestri di canne»». ${ }^{32}$ In ogni caso, al di là dei singoli provvedimenti presi congiuntamente dai due Rettori, in quell'accenno a quella cosa tanto necessaria in ogni stato si intravede la penna del Capitano: la coscienza dello stato territoriale e dell'importanza per esso della dimensione militare sarà uno dei leitmotiv dei Discorsi Politici, che Paruta andava stendendo proprio fra gli anni Ottanta e gli anni Novanta. ${ }^{33}$

Ancor prima, in un dispaccio del giugno 1591 Morosini e Paruta dichiarano di essersi messi a investigare le cause dell'emigrazione di molte famiglie di produttori di armi della Val Trompia ${ }^{34}$ verso lo Stato di Milano, nata secondo loro dalla «prohibitione di non poter estrarre Canne che non siano incassate». Così molti maestri, «pensandosi di provedere a questo danno» e «tirati da' partiti più avantaggiosi, che loro sono stati proposti», hanno lasciato le valli bresciane per territori esterni al dominio della Serenissima. ${ }^{35}$ Già in quest'analisi si può

31 «Saprà dunque Vostra Serenità questa materia della ferrarezza essere al presente ridotta a così grandi et così manifesti disordini che si può affermarle con verità che, non se le dando presto alcun rimedio, venirà in breve tempo più presto a mancare del tutto che a declinare tale arte in questa Città, con gran deterioramento de' suoi datij, con danno notabile di questo popolo et, ciò che più importa, con ridursi a mancamento di quella cosa che è tanto in ogni stato necessaria, et della quale questo paese è stato particolarmente gratiato dalla natura, cioè delle arme da offesa et da difesa» (Senato Terra, filza 121, Dispaccio dei Rettori di Brescia, 04/05/1591, c. 1r). Vd. anche: «la materia delle armi è tanto in ogni Stato necessaria, et alla provisione della quale deve ogni Prencipe per tempo provedere» (Senato Terra, filza 121, Dispaccio dei Rettori di Brescia, 12/07/1591).

32 Barbiroli 2012:30. Per la storia di questo fontego vd. Morin/Held 1980:56-66

33 Su questo aspetto dei Discorsi Politici vd. GIANI 2016.

34 Per un breve profilo storico-economico della Val Trompia nella prima Età Moderna vd. MoRIN/HeLD 1980:16-21.

35 «noi siamo molto ben certificati che da poco tempo in qua, et sotto il nostro medesimo Reggimento, si sono levate da Valtrompia molte fameglie de quelli che 
apprezzare la chiarezza e l'acutezza di una vera e propria scienza di governo dei rettori veneziani, fondata sulla ricerca delle cause effettive e profonde dei fenomeni (in questo caso economici) ${ }^{36}$ e indirizzata ad una conseguente proposta di soluzione:

parer nostro saria che, per proveder che questo disordine non vada più inanti, et quest'arte, con la quale solevano viver numero grande di persone, adoperandosi in essa in più sorte d'essercitij, non resti del tutto destrutta et annihilata, che si dovesse, levando questa prohibitione, lassar correr questa mercantia, come per inanzi soleva ${ }^{37}$

Il parer dei rettori, insomma, è un'opinione sì personale, ma ben

lavorano in quest'arte di Canne [d'Archibusi], et sono andati ad habitar ne lo stato di Milano, et altrove; di che havendo noi voluto investigare le cause, troviamo una, c'assai principale essere questa prohibitione di non poter estrarre Canne che non siano incassate. Però che i mercanti che vogliono condur archibusi in paesi lontani convengono sentire molta spesa et incommodo non solo per essere l'imboglio molto grave, ma anco perché le casse nei longhi viaggi restano fracassate; onde è nato che, pensandosi di provedere a questo danno, si sia data occasione d'introdurre molte fucine, che non solevano essere prima, ne lo stato di Milano, in Toscana, nel Regno di Napoli, in Saluzzo, et in altri luochi, ove sono concorsi molti maestri, tirati da' partiti più avantaggiosi, che loro sono stati proposti» (Senato Terra, filza 121, i Rettori di Brescia, 07/06/1591, c. 1r). Interessante notare come l'emigrazione degli armaioli bresciani in territori economicamente più proficui per la loro attività sia fenomeno ricorrente: per il primo Cinquecento vd. LANARO 1999:74; per una prospettiva temporalmente più ampia, BARBIROLI 2012:26. Sul problema dell'emigrazione dei produttori di armi a causa del ciclico ricorso, da parte della Serenissima, ad una «cieca politica di crudo fiscalismo e stretto protezionismo», vd. PASERO 1940:288. Per un profilo storico della produzione delle canne delle armi da fuoco in Val Trompia (nonché per alcuni assaggi di lessico delle armi da fuoco a questa altezza cronologica) vd. MoRIN/HeLd 1980:22-38.

$36 \mathrm{Vd}$. anche: «conoscendo, et per la qualità della cosa, et per l'informatione commessane più d'una volta da Vostra Serenità, che la materia dell'armi sia grandemente stimata» (Senato Terra, filza 121, 23/10/1591, c. 1r). Recentemente, Simone Signaroli ha mostrato, nel confronto fra il francese Isaac Casaubon e il veneziano Domenico Molin (di una generazione più giovane del Paruta) una significativa differenza di approccio di fronte ai testi antichi: in particolare, del secondo viene ricordata una «una notizia allora inedita riguardante $\mathrm{i}$ trattati trecenteschi sul commercio del sale tra Venezia e Brescia recuperata nell'archivio della cancelleria secreta di Palazzo Ducale» (a cui il Molin aveva accesso in quanto patrizio) per sottolineare come all'erudizione del francese corrispondesse un interesse che nel veneziano ai agganciava «alla propria condotta di uomo politico» (SIGNAROLI 2017:25).

37 Senato Terra, filza 121, i Rettori di Brescia, 07/06/1591, c. 1 r. 
motivata e razionale, frutto di un'analisi quanto più possibile oggettiva del disordine in questione. Quando poi i Rettori stessi illustrano la possibile obiezione di «alcuni pochi maestri che si adoprano nel far le casse», ${ }^{38}$ la conclusione («né è però in alcun modo paragonabile il commodo de questi pochi allo incommodo e interesse de molti») $)^{39}$ richiama un principio cardine della politologia parutiana, quello della superiorità del bene comune rispetto al bene del singolo quando si tratta di una civitas. ${ }^{40} \mathrm{Il}$ Senato veneziano in effetti, in data 24 settembre 1591 revocherà la parte del $1588^{41}$ che prevedeva la probibitione; ${ }^{42}$ a inizio novembre Morosini e Paruta saranno lodati per la gestione del negotio del fabricar arcobusi, gestione che «mostra chiaramente la vostra molta diligenza in tutte le cose di servitio publico». ${ }^{43}$

\subsection{3.«Biave» e carestia}

Gli anni dell'incarico bresciano furono fra i peggiori del decennio per quanto riguarda la penuria alimentare: il 1591, in particolare, è ricor-

38 Gli incassatori erano i falegnami che univano canna e piastra metalliche alla cassa, composta da fusto, impugnatura e calcio; a differenza dei tedeschi, gli incassatori bresciani producevano casse alla lombarda, cioè a fusto incurvato. Su questi artigiani vd. GAIBI 1964:878-879.

39 Senato Terra, filza 121, 07/06/1591, c. 1v.

40 Su tale aspetto del pensiero politico parutiano vd. GIANI 2014B.

41 Nel 1588 «il Senato, per tentare di ovviare ai segni del declino manifestati dall'industria armiera [della Val Trompia], stanziò un prestito straordinario di ben trentamila ducati al beneficio della stessa [...] Nella medesima disposizione, con l'intento di giovare ai fabbricanti di corazze (allora ancora chiamati "armaioli") e ai maestri da casse a Brescia, fu vietata l'esportazione del ferro da armatura e delle canne non incassate. Naturalmente quest'ultima misura si rivelò subito disastrosa per le maestranze gardonesi. I mercanti stranieri e gli esportatori italiani trovarono intollerabili le maggiori spese di trasporto e imballo delle armi incassate e complete, le quali erano molto più voluminose e pesanti delle sole canne, ed anche più fragili» (MORIN/HELD 1980:54). Nei dispacci dei Rettori c'è un richiamo al prestito statale del 1588, fatto «per solevar l'arte del fabricar arcobusi, la qual si vedeva in questo paese andar mancando» (Senato Terra, filza 121, 23/10/1591, c. 1r).

42 Vd. l'accenno a questa decisione del Senato in MORIN/Held 1980:61.

43 Senato Terra, filza 121,02/11/1591. A metà novembre i Rettori scrivono che circa la questione del «fabricar Canne d'archibusi» s'è passati ai fatti: quindi «da noi è stato trattato et concluso un partito di far fare cinquantamille Canne d'archibusi nel tempo et modo che dalli Capitoli che li mandiamo qui inclusi ella potrà vedere» (Senato Terra, filza 122, 19/11/1591). 
dato come anno di «crisi e carestia». ${ }^{44}$ Della questione delle biave in realtà doveva occuparsi personalmente (e si occupò, in effetti, almeno stando ai dispacci $)^{45}$ il podestà Morosini, non il capitano Paruta, che pure qualche anno prima (nel gennaio 1587, per la precisione) aveva ricoperto l'incarico di Provveditore sopra le biade. ${ }^{46}$ Eppure, anche in qualche dispaccio fir-

44 AAVV 1978:154.

45 In un dispaccio firmato a quattro mani il 17 settembre 1591, è il Morosini a prendere esplicitamente la parola, essendo stato accusato di rappresentanti della Val Trompia e della Val Sabbia di aver ignorato le loro lettere di agosto in cui chiedevano aiuto per la mancanza di biave. Il Podestà si difende dicendo di non averle ricevute, altrimenti avrebbe sicuramente risposto, «sì come ho avuto sempre desiderio di dovenir esse valli di biave ne' maggiori suoi bisogni», «dovendo havere però principalmente consideratione alla strettezza de formenti nella quale hora si trova questo territorio per il cativo raccolto passato» (Senato Terra, filza 122, 17/09/1591). L'inverno precedente doveva essere stato orribile, almeno a quanto raccontato da Morosini in un suo dispaccio: «io mi trovo di sì fatta maniera aggravato di convenir sostentare un numero tale di Popolo in tempo spaventoso, com'è questo, et per il mancamento delle biave, et per le importanti conseguenze de molti mali che soprastano, et che si sogliono commettere da quelli che sono cacciati dalla fame» (Capi di X, 17, 13/11/1590, cc. 1v-2r). Il Morosini parla della questione delle biave in molti altri dispacci, fra cui vale la pena di segnalare quelli del 14/09/1591 (contenuto in Capi di X, 32), 21/09/1591 (Capi di X, 35) e 15/01/1592 (Capi di X, 46b). Riguardo il problema dell'approvvigionamento alimentare della Val Trompia in età moderna si ricordi come «nelle relazioni e nei dispacci dei Rettori veneziani viene sempre sottolineato che la natura sterile ed aspra della Valle era in grado di fornire "vittuarie" per la popolazione residente bastanti solo per 2/3 mesi l'anno. Il resto veniva importato dalla pianura» (MORIN 1981:69).

46 Per una visuale su tutti gli incarichi annonari ricoperti dal Paruta nella sua carriera vd. GIANI 2013/2014:386-395. Rimando allo stesso lavoro per una bibliografia sulle carestie degli anni Novanta e sulla loro gestione da parte della Serenissima. Al riguardo, va segnalato come le fonti archivistiche consultate per questo lavoro contengano molto materiale interessante. Come esempio, si prenda questo passaggio: «La nave fiamenga chiamata Lion Negro, patron Ghielmo de Nicolò, che venne carica di formenti in questa Città, partita con il carico del tratto di essi formenti di ... per Fiandra; è stata nel stretto di Zibilterra dal General delle Galere di Spagna retenuta con altre Navi. Onde, sapendo noi Mercanti et asseguratori di questa piazza interessati in essa, et tale retentione è indebita prima perché è vassillo fiamengo, poi perché sta un editto di Sua Maestà Catholica che tutte le Navi che conducono formenti in Italia siano libere di andar et di ritorno, humilmente la [= il Doge] supplicano che si degni scrivere all'Illustrissimo Ambasciator suo residente presso Sua Maestà ... per la ricuperatione della suddetta Nave, nella quale si tratta dell'interesse dei suoi Cittadini et sudditi, et saria in conformità del decreto et salva condotto da Lei fatto in generale a tutte esse Navi, et come ... sudetta sarà caricata di formenti per Italia, [...]» (Senato Terra, filza 121). 
mato a quattro mani, si parla di questo problema. Nel novembre del 1591, ad esempio, Morosini e Paruta non solo inoltrano la supplica del Territorio di Brescia per avere una dilazione di pagamento del sussidio, ma l'appoggiano pure, «vedendo che tuttavia continua la mala conditione de gli anni penuriosi»; così «crediamo che sarà effetto proprio della sua benignità se anco in questa occasione questi suoi fidelissimi populi sentiranno il frutto della gracia di Vostra Serenità». ${ }^{47}$ Del resto, la preoccupazione non solo per il benessere materiale dei sudditi ma anche per la pace sociale e l'obbedienza verso le autorità era largamente condivisa a Venezia, come si può apprezzare dalle risposte inviate ai rettori bresciani. ${ }^{48}$

\subsubsection{Liti nobiliari e fazioni}

Un altro problema nel quale Paruta venne implicato fu quello legato ai litigi fra le famiglie nobili locali («li Bresciani sono sì fatti, pieni di superbia e di svampolamenti», scriveva il vescovo Domenico Bollani - personaggio della Perfettione parutiana - nel 1577) ${ }^{49}$ come documentato dal fondo delle lettere dirette dai Rettori ai Capi del Consiglio di $\mathrm{X}$, nel quale vi sono numerose missive firmate insieme da li Rettori $\mathrm{di}$ Brescia. ${ }^{50}$ A fine novembre 1591 è lo stesso Paruta ad essere interpellato da un nobile locale, il quale sarebbe «venuto [...] al Palazzo di me Capitano per trattare sopra a questo $»^{51}$ (laddove il Palazzo è da identifi-

47 Senato Terra, filza 122, Tommaso Morosini e Paolo Paruta, da Brescia, 19/11/1591. Per altro, si ricordi come il Magazzeno delle Biave fosse all'epoca ospitato proprio all'interno dell'attuale Broletto: PANAZZA 1964/1965:194.

48 In una missiva dell'agosto del 1591 i Savi del Consiglio, coscienti della la calamità della Val Trompia, ordinano ai due Rettori i Brescia di aiutare i valligiani permettendo loro l'estrattione delle biave, spaventati dalla «temeraria rissolutione di quei principali di Valtrompia di mettere insieme gente armata per haver per forza ad ogni prezzo formenti et levar da carri quei che venivano condotti in quella Città per farne particolarmente mercantia, sì come è stata a noi di somma molestia, così ci ha mossi a dirvi con il Senato che $[. ..] \gg$ (Senato Terra, filza 122, 17/08/1591)

49 Cit. in Gamba 2016:73.

50 Capi di X. Non essendo segnalate nella voce biografica di Benzoni, elenco quelle firmate dai Rettori (con voce comune, o con le due firme). Si tratta delle u. c. 27 (10/06/1591), 33 (02/04/1591), 35 (21/09/1591), 37 (22/09/1591), 39 (27/10/1591), 41 (24/11/1591), 42 (25/11/1591), 43 (21/12/1591), 44 (01/01/1592), 45 (08/01/1592), 48 (02/03/1592), 52 (20/05/1592), 53 (04/06/1592).

51 Capi di X, 41, c. $1 \mathrm{r}(24 / 11 / 1591)$. 
care con il Broletto). La lite fra i membri di alcune famiglie nobili quali gli Avogadro, i Martinengo e i Calino viene illustrata ai Capi di X, ma subito dopo minimizzata:

nel resto, levata questa occasione, le cose di questa Città passano con tanta quiete, che nel tempo di questo nostro Reggimento non ha, si può dir, sentito alcun altro travaglio fuorché questi rumori, i quali però, per gratia del Signor Nostro Dio, fin'hora non hanno partorito alcun de quelli inconvenienti che si temeva, ma de' quali si sta però ancora in molto pericolo ${ }^{52}$

Le speranze dei Rettori, purtroppo, vengono deluse, tanto che in un dispaccio del giorno seguente sono costretti a narrare la brutta piega che gli eventi hanno preso a Brescia. Dopo aver parlato delle gesta dei protagonisti, Morosini e Paruta sottolineano le gravità della questione particolare rispetto alla pace sociale della città intera:

mantenersi e fomentarsi le difficultà di questa pace anco da altri che scopertamente non se ne mostrano interessati, e farsi confini non pur dannosi alla quiete della Città per quello che hora si vede, ma per cose maggiori di tenervi assolutamente la maggioranza, quando una di queste fattioni, così antiche et così potenti in questa Città, per quelli accidenti che apportano queste lunghe inimicizie, venisse a debilitarsi et a mancare de Capo de auttorità. Il che, come può esser bene inteso dalle Vostre Illustrissime Signorie, così crediamo ch'elle giudicheranno di molto servicio publico il porvi in qualche modo la sua man et la sua auttorità, perché queste famiglie principali, cessati questi rumori, si mantenghino nell'essere e stato che si ritrovano, e sia levata l'occasione del far sette et nutrir humori sempre poco utili, et per la quiete de' populi, et per gli interessi de' Prencipi ${ }^{53}$

Il lessico della citazione appena riportata è in assoluta continuità non solo con quello dei rettori veneti a Brescia, ${ }^{54} \mathrm{ma}$ - quel che più

52 Capi di X, 41, c. 1v (24/11/1591).

53 Capi di $X, 42$, c. $1 \mathrm{r}(25 / 11 / 1591)$.

54 Un esempio anteriore ed uno posteriore. Il capitano di Brescia Daniele Priuli, nel 1585, parlava esplicitamente di «fattioni Avogadresca» e «Martinenga»: vd. Manno 1987:262. Nella Relazione del capitano Giovanni Paolo Gradenigo, datata gennaio 1607, si dirà che «sono li principali [fra gli abitanti di Brescia] divisi in fattione, [...] et dividendo anco tutti gli altri, che dipendeno secondo i loro particolar affetti, chi 
importa - con quello dei Discorsi Politici parutiani, in particolare con quelli che trattano appunto il problema delle fazioni e di coloro che, singoli o famiglie che siano, riescono la conquistare la maggioranza (non in senso elettorale, ma come 'egemonia di fatto') all'interno di una comunità politica. ${ }^{55}$ Ciò che è da rilevare è appunto come il Paruta capitano da Brescia utilizzi, per parlare degli Avogadro o dei Martinengo, le stesse parole-chiave che nell'opera politologica verranno usate per Giulio Cesare nella Roma tardo-repubblicana o per i Medici nella Firenze rinascimentale.

Da parte loro, nel prosieguo del dispaccio, i Rettori provano a delineare una loro linea di intervento possibile (l'immagine della bilancia, altra key-word del lessico politico parutiano), ${ }^{56} \mathrm{ma}$ in un clima di sconforto reso evidente dalla chiusa:

Dal canto nostro siano Vostre Illustrissime Signorie sicure che non si manca di star sempre avertiti per tener giusta la bilancia, et perché né segua al presente alcun scandalo, né si apra la strada ad altri maggiori, che potrebbono seguire per l'avenire. Tuttavia gli ordini di questa Città si trovano in modo contaminati da questo affetto delle fattioni che, dovendo anco esso Conte Carlo [Martinengo] riddursi ad habitar in questa Città, vediamo difficile il proveder ad ogni disordine. Et da gli tanti officij fatti per noi per la conclusione della Pace, fin'hora non veggiamo di poterne sperar alcun buon frutto ${ }^{57}$

da una d'esse fattioni et chi dall'altra, si nutriscono le continue nemicitie, multiplicano i homicidij, non mancano $i$ assasinamenti, et concorrono in gran numero a tante iniquità li più scelerati siccarij et assassini, venendo esser protetti et favoriti nella propria casa dei principali, che, fomentando in questa maniera col dispendio, si può dire, di tutte le lor facoltà, attioni così inhumane et diaboliche, riesce quella patria da questo capo veramente infelicissima» (cit. in TAGLIAFERRI 1978:169-170). Sullo «spirito di prepotenza» che «animava la nobiltà locale» di Brescia a fine Cinquecento vd. MAZzOLDI 1964:3-12.

55 Per il significato di fazione e di maggioranza nel lessico politico parutiano vd. GIANI 2012:129 e GIANI 2014:182.

56 GIANI 2012:188-191.

57 Capi di X, 42, c. $1 v$ (25/11/1591). In un successivo dispaccio del $1^{\circ}$ gennaio 1592 i Rettori dichiarano di aver notificato a Francesco Avogadro, Carlo Martinengo e Vincenzo Calino la decisione delle autorità, ossia il trasferimento a Venezia: Capi di X, 44. Troveremo membri di queste tre famiglie fra gli arrestati del febbraio 1599 , allorquando il Consiglio dei Dieci interverrà direttamente per fermare la faida nobiliare che stava insanguinando la città e che pareva essere uscita fuori da qualsiasi controllo: vd. 


\subsubsection{Fortificazioni}

Un ulteriore importante compito del capitano di Brescia era quello riguardante la cura della fortificazioni presenti sia in città sia nel Bresciano. ${ }^{58}$ Tale aspetto è per altro collegato a quello appena presentato dell'ordine interno: si ricordi ad esempio l'immagine del freno e della chiave usata qualche anno prima da Paolo Contarini per spiegare che il castello di Brescia era utile non solo per i nemici esterni ma anche per chiunque, all'interno della città, avesse voluto machinare contro il potere costituito di Venezia. ${ }^{59}$

Paolo Paruta, da canto suo, non era nuovo a questo tema, non solo per le cariche politiche ricoperte, ma anche per un suo perspicuo interesse teorico che poi si concretizzerà nelle pagine dei postumi Discorsi Politici (soprattutto in uno dei più riusciti, ossia il Discorso sulle Fortezze) ${ }^{60}$

In città, il capitano dovette prima di tutto occuparsi di quel castello sulle cui vicende egli doveva essere informato da tempo, visto che qualche anno prima troviamo anche la sua firma (nelle vesti di Savio di Terraferma) nella famosa parte dell'estate 1588 che risolse i molteplici dissidi che rischiavano di far naufragare il cantiere bre-

MAZZOLDI 1964:6-7. Per l'espressione tener giusta la bilancia (ed analoghe) nel lessico politico parutiano, vd. GIANI 2012:539.

58 PASERO 1940:12. Come caso esemplare vd. le varie figure di capitano citate in Manno 1987, saggio dedicato al restauro del Castello di Brescia negli ultimi due decenni del XVI secolo. Sulla storia cinquecentesca dell'edificio, vd. MARCHESI 1984:163-166.

59 Nel 1584 il provveditore generale di Terraferma Paolo Contarini, affermando che Brescia si trovava «in molta confusione per diversi humori che correno», proponeva di aumentare $\mathrm{i}$ soldati in città, osservando anche che non era bene «tenere il castello, che è freno et chiave d'ogni cosa, nel termine che si trova al presente, per non dar causa ad alcuno de machinarle contro». Antonio Manno, collegando l'immagine del freno e della chiave ad alcuni eventi di quegli anni, ha sottolineato il doppio piano dell'immagine: il castello quindi per controllare non solo per la difesa esterna ma anche per l'ordine pubblico interno: vd. MANNO 1987:259-260.

60 DP II.VIII. Su questo discorso vd. il mio paper Venetian warfare in the age of Palmanova: Paolo Paruta's Discorsi Politici (1599), presentato in data 18/11/2011 all'interno della conferenza londinese «New Research in Military History» (organizzata dalla British Commission for Military History), il cui testo è disponibile all'indirizzo: https://www.academia.edu/1888672/Venetian_warfare_in_the_age_of_ Palmanova_Paolo_Paruta_s_Discorsi_Politici_1599_[Ultimo accesso: 07/10/2017]. 
sciano. ${ }^{61}$ All'inizio del 1591 (sotto quindi il capitanato di Paruta), la serie di «cedimenti verificatisi lungo le muraglie appena costruite» raggiunse

proporzioni preoccupanti, tanto da far chiamare sul luogo per una perizia gli ingegneri Malacreda e Lorini. Solo in dicembre, sentiti anche i due proti inviati da Venezia e sottoposti i loro pareri a del Monte e Savorgnan, si decreta la costruzione di un muro di sostegno alto venti piedi per impedire alle muraglie di crollare sotto il proprio peso ${ }^{62}$

Visti questi problemi strutturali, nel maggio del 1591 Giulio Savorgnan, allora Soprintendente generale delle artiglierie e delle fortezze, ${ }^{63}$ venne «inviato a Brescia per esaminare i cedimenti delle mura». ${ }^{64} \mathrm{Si}$ tratta di un'informazione straordinariamente importante, perché permette di documentare il fatto che Paruta, nelle vesti di capitano di Brescia, ebbe modo di vedere in azione direttamente sul campo il celebre architetto militare: così, la lode che egli ne fece nella primigenia versione del suo Discorso sulle Fortezze $e^{65}$ acquista una concretezza fino ad ora non investigabile.

Spostandosi da Brescia al Territorio, ci si imbatte in una seconda testimonianza, questa volta proveniente da una fonte edita, ossia l'opera storiografica dedicata da Domenico Codagli alla storia di Orzinuovi. Il libro, stampato a Brescia da Giovan Battista Borella nel 1592, si conclude col racconto dei tre grandi eventi accaduti nella città natale del Codagli nel 1591, l'ultimo dei quali è proprio la visita del Paruta con occasione di rivedere la militia. Questo l'estratto:

61 MANNO 1987:273.

62 MANNO 1987:278

63 Su questa carica e sul suo valore politico vd. CASELLA 2009:2268.

64 MANNO 1987:278

65 In un suo lavoro del 1986 Antonio Manno ha rivelato un'importantissima variante presente in un testimone manoscritto del Discorso delle Fortezze oggi conservato presso l'Archivio di Stato di Venezia: in un passaggio poi cassato nella versione a stampa, Paruta lodava «l'illustrissimo Signor Giulio Savorgnano generale delle fortezze della Repubblica di Venezia, il quale con certissime raggioni procedendo nel fabricare fortezze leva ogni dubbio, che altri potesse havere» (cit. in MANNO 1986:132). Tale variante è presente in molti altri testimoni manoscritti: alla censura di questo passo (da inquadrare dentro i rapporti politici intrecciati negli ultimi anni di vita da Paruta) dedicherò un lavoro specifico. 
quella felicissima giornata, non sia da tacersi, quando, a prieghi di questa spettabile Communità, lasciatosi vincere il grand'Oceano delle Cortesie et delle leggiadre Historie, Paolo Paruta, di nome, di sangue, di valore et d'opre Illustrissimo, al presente Historico in luogo del Contarini per la Republica, et vigilantissimo Prefetto di Brescia, (che fora per eternar' di gloria più che d'inchiostro, con l'aurata penna del suo terso e facondo scrivere, il sempre Augusto e invittissimo sangue Veneto) non nata a pena la soavissima stagione del pomifero autonno, con occasione di rivedere la militia, gionse insino a gl'Orci, dove, con apparati degni e convenevoli, fu da gl'Orceani molto accarezzato. Gionsi io fra tanto (quasi che all'apparir d'un Prot'historico et tanto gran scrittore, non fussi più degno di repigliar la penna) in fine di questa Historia ${ }^{66}$

Per ragioni cronologiche, la visita del Paruta (che, in quanto capitano di Brescia, doveva aver cura non solo delle fortificazioni, ma anche delle milizie dislocate lungo tutto il Territorio) ${ }^{67}$ andrà collocata nel pomifero autonno del 1591, l'unico che l'Historico per la Republica di Venezia (subentrato nel febbraio 1580 al defunto Alvise Contarini, Storiografo Pubblico dal marzo 1577) ${ }^{68}$ trascorse a Brescia. ${ }^{69}$ Le lodi del Codagli ${ }^{70}$ non solo al politico ma pure al tanto scrittore fanno compren-

66 CODAGLI 1592:205-206.

67 In molti dei dispacci oggi conservati nell'Archivio di Stato di Venezia è documentato come i Rettori di Brescia si preoccupassero dello stato della cinta muraria di Orzinuovi (su cui vd. le immagini presenti in MARCHESI 1984:152). Quest'ultima era stata effettivamente rimaneggiata di continuo fra metà Quattrocento e i primi anni del Cinquecento: vd. LANARO 1979:XXV-XXVI. Si ricordi come Orzinuovi avesse strettissimi legami anche economici con Brescia, i cui ricchi cittadini possedevano molti dei fondi locali: vd. LANARO 1979:XXI.

68 Cozzi 1963/64:56, che giustamente puntualizza come la data del documento riportato dallo Zeno (ossia «18.02.1579») vada letta more veneto: capita infatti ancora di leggere, in qualche contributo contemporaneo, che Paruta venne eletto Storiografo Pubblico nel febbraio 1579 (ipotesi non solo in contraddizione coi documenti superstiti, ma con la stessa logica, visto che Alvise Contarini morì nel novembre del 1579). L'articolo di Cozzi riporta ampi stralci delle lettere d'incarico del Contarini, del Paruta e del successore di quest'ultimo, ossia Andrea Morosini; lo Zeno aveva riportato per intero quelli del Contarini e del Paruta (ZENO 1718:XVIXVII). Oltre ai documenti archivistici citati da Cozzi segnalo due copie delle parti del Contarini e del Morosini conservate in VenEZIA, Archivio di Stato, Riformatori allo Studio di Padova, 363

69 L'autunno in questione non può essere quello del 1592 perché la lettera dedicatoria dell'autore è datata «Giovedì Santo del 1592» (CODAGLI 1592:7).

70 Il Codagli, in quel momento residente nel convento domenicano veneziano 
dere come Paruta fosse visto dai contemporanei spesso in questi due aspetti della sua personalità (come vedremo nell'iscrizione del Broletto), nonché le grandi aspettative del pubblico per la Historia Vinetiana $^{71}$ che purtroppo vedrà la luce solo nel 1605, ben sette anni dopo la morte dell'autore.

\subsubsection{Ulteriori questioni}

Come accennato, Paruta venne sicuramente implicato nella risoluzione di altre questioni aperte in quegli anni a Brescia, che tuttavia in questa sede non possiamo che riassumere, fornendo tuttavia i rife-

di San Domenico in Castello, aveva avuto probabilmente l'occasione di apprezzare l'opera storiografica del Paruta grazie ai numerosi estratti clandestini che circolavano in quel momento a Venezia (per un esempio di questa circolazione si ritorni alla vicenda del Riccoboni, su cui vd. FAVARO 1891). Se dall'ambiente domenicano ci spostiamo a quello francescano, si noti come nel Libro Secondo del dialogo Lo Scolare (Venezia, 1588), Bartolomeo Meduna includa Paruta in una lista come la seguente «furono valent'huomini, dotti et universali, come oggi sono parimente Francesco Piccolomini, Tomaso Contarino e Polo Paruta, Marc'Antonio Mocenico e Scipion Gonzaga» (p. 84). Sulla lunga durata della stima che molti storiografi d'area veneta avevano del Paruta si veda la curiosa testimonianza riportata recentemente alla luce da Galtarossa. Quasi due secoli dopo la morte di Paruta, nel 1770, il Cancellier Grande Giovanni Colombo, interrogato dai Capi del Consiglio dei X su come migliorare l'insegnamento ai futuri componenti della Cancelleria ducale, suggerì di passare, come testo per l'esame di lettura, dai classici latini ad un qualche «"buon libro italiano" a stampa, e in particolare propose la Storia veneta del Paruta, alternando l'utilizzo ora del primo e ora del secondo tomo, oppure di qualche altro libro scritto in "buona lingua italiana"(...)» (cit. in GALTAROSSA 2013:86).

71 Per un breve profilo di quest'opera vd. GIANI 2012:68-72. La spasmodica attesa per la pubblicazione si trascinerà anche oltre il 1598, anno di morte dell'autore. Nel 1603, ad esempio, Leonico Goldioni, nella sua edizione de Le cose maravigliose et notabili della Città di Venetia scriveva così: «Paolo Paruta Cavalier, et Procuratore per dottrina ammirabile, et che nel negotiar per la Republica hebbe pochi pari, onde in età ancora giovenile fu adornato del titolo dignissimo di Procuratore, ha scritto l'historia della sua Republica che tosto si daranno (come si spera) alla Stampa: et pur si veggiono stampate diverse cose delle sue; et della perfettion della vita Politica, et fe' discorsi nella materia di Stato, et altre cose che 'l dimostrano singolare, et che era degno di ogni honor più honorato» (p. 183). La situazione editoriale delle opere parutiane disponibili in versione a stampa è effettivamente quella del 1603: sul mercato editoriale veneziano erano disponibili solo la Perfettione della Vita Politica e i Discorsi Politici - la Historia Vinetiana sarebbe stata pubblicata a cura degli eredi Paruta da lì a due anni. 
rimenti archivistici e/o bibliografici utili per ulteriori ricerche: la pubblicazione dell'estimo della città di Brescia e del suo Territorio ${ }^{72}$ la collaborazione con le autorità del Ducato di Ferrara per la cattura di un pericoloso bandito (suddito veneto) che continuava a sconfinare nei territori estensi, ${ }^{73}$ una regolamentazione per la risoluzione delle cause giudiziarie, ${ }^{74}$ questioni fiscali varie (nelle quali è ragionevole supporre che Paruta, viste non solo le sue esperienze amministrative passate in campo economico ${ }^{75}$ ma soprattutto le sue prerogative presenti di capitano ${ }^{76}$ fosse più implicato del Morosini) ${ }^{77}$ suppliche di singoli sudditi. ${ }^{78}$

72 In un dispaccio del dicembre 1591 i rettori, annunciando la conclusione, con molta solennità publicata, dell'«Estimo Generale tra questa Magnifica Città et il Territorio» incominciato ben 28 anni prima, sottolineano che la sodisfattione universale è ben giustificata, visto che questo nuovo strumento amministrativo «leverà le occasioni di molte contese tra essa Città et il Territorio, et quelli ch'erano indebitamente aggravati se sentiranno il debito solevamento» (Senato Terra, filza 122, 12/12/1591).

73 Documento datato 22 giugno 1591, cit. in LAVEN 1994:232. Sulla genesi di tali banditi, spesso provenienti dalle litigiose famiglie nobili bresciane, vd. MAZZOLDI 1964:4.

74 Dispaccio dei Rettori, da Brescia, 15/01/1592 (Senato Terra, 122). Si tratta della missiva citata nel documento veneziano del 22 febbraio 1592 (riportato da MAZZuCHELli 1732:72) in cui si parla degli «Illustrissimi Signori Tommaso Morosini Podestà, e Paolo Paruta Capitanio degnissimi Rettori di questa Città, e suo Distretto».

75 Nel 1580 Paruta era stato eletto Provveditore alla Camera degli Imprestiti, nel 1585 Savio alla Mercanzia: vd. BENZONI 2014; circa la sua personale esperienza di mercante privato durante gli anni Sessanta vd. invece GIANI 2013/2014:392.

76 Per il rapporto fra il capitano di Brescia e la Camera Fiscale della città vd. PASERO 1939:13.

77 In una lettera indirizzata ai Rettori di Brescia, il Senato risponde positivamente circa la «persona et denontiante secreto che si offerisce far ricuperare a quella Camera un credito stimato del tutto inesigibile di ducati 4000 incirca», ma senza far sapere niente a funzionari della Camera stessa (Senato Terra, filza 121, 12/09/1591). Tre mesi dopo, Tommaso Morosini e Paolo Paruta spiegano alle autorità veneziane la questione di Giovan Maria Bonvicino: avendo in appalto un datio, era stato accusato di trattenerne illecitamente una parte, e quindi aveva avuto a che fare con la Camera fiscale (Senato Terra, filza 122, 17/12/1591 m.v. = 1592).

78 Nel maggio 1592 i Rettori inoltrano al Senato la supplicatione di Ottavio Luzzaghi, che ha 12 figli e una moglie ancora abbastanza giovane, «i quali figli si affatica di allevare virtuosamente, mantenendoli in Academia, et non sparagnando ad alcuna spesa o fatica per allevarli bene» (Senato Terra, filza 121, Tommaso Morosini e Paolo Paruta, 12/05/1592). 


\section{Paolo Paruta e il Broletto di Brescia}

\subsection{Il capitano e i lavori per il Broletto}

Per quanto mai messo in luce esplicitamente nei vari studi sulla storia del Broletto, Paolo Paruta ebbe un piccolo ruolo nella ricchissima storia ${ }^{79}$ di questo edificio,

che è - contemporaneamente - accumulo di stratificazioni architettoniche depositatesi per almeno otto secoli; elemento cardine ma non unico sito caratterizzante di un tessuto urbano articolato e ricco; luogo simbolico nei tempi lunghi della storia del potere politico, municipale, territoriale, statale. La storia ha "costruito" il Broletto che oggi vediamo ${ }^{80}$

Come noto agli storici dell'architettura, i due rettori veneti a cui vanno ascritti i lavori più massicci sull'edificio in epoca moderna (rispettivamente nello scorcio finale del Cinquecento e soprattutto nel primo Seicento) sono il Bernardo e il Da Lezze. ${ }^{81}$ Eppure, incrociando la cronologia dell'incarico parutiano con le date riportate dai documenti, possiamo sicuramente attribuire a Paruta almeno due decisioni.

La prima (ragionevolmente, presa in accordo col podestà Morosini) è quella, datata 22 gennaio 1592, «di unire la casa del Podestà a quella del Capitano in Broletto e vendere l'antico palazzo del Carmagnola (palazzo Vecchio)». ${ }^{82}$ Le sedi due rettori erano state fino a quel momento divise, ma era ormai tempo di mettere in atto questa razionalizzazione degli spazi pubblici del potere veneto in città. ${ }^{83}$

79 Oltre ai vari lavori specialistici che cito in nota, segnalo, come buon punto di partenza sull'argomento, il sintetico RAPAGGI 2012.

80 ZANE 1998:2. Per un profilo dei luoghi del potere veneziano a Brescia nella prima parte del Cinquecento vd. Bowd 2010:86-90.

81 Piovanelli 1975:30, CocColi / SCALA / TRECCANI 2009:112.

82 VOLTA 1987:22. A partire dal 1593 il Podestà si trasferì effettivamente ad abitare in Broletto, dove già risiedeva il Capitano: vd. VolTA 1986:68.

83 Nel corso del XVI secolo «il Broletto «divenne sede dei due Rettori che rappresentavano il potere della Serenissima: ovvero il Podestà, con mansioni politiche, amministrava la giustizia ed aveva autorità simile a quella degli attuali prefetti, e il Capitano, che rivestiva la massima carica in campo militare, economico-fiscale e con il Podestà amministrava la giustizia» (COCCOLI / SCALA / TRECCANI 2009:112). La decisione era significativa perché «per tutto il Cinquecento Venezia non prestò grande 
La seconda decisione (conseguenza logica della prima) venne invece presa da un capitano che per motivi cronologici deve essere identificato con Paruta: «il 6 marzo 1592 si concretizzano gli accordi per ricongiungere gli uffici dei due Rettori Veneti nel Broletto: accordi dettati dal Capitano a Dionisio Bolda, ingegnere militare, dipendente diretto della Serenissima, mandato già nelle fortezze della Terra Ferma Veneta». ${ }^{84}$

Paruta, comunque, non farà in tempo a vedere la realizzazione dei lavori, che avverranno solo a partire dal $1593,{ }^{85}$ quindi sotto il capitanato del suo successore.

\subsection{Paolo Paruta e gli edifici pubblici}

Non era la prima volta che l'autore della Historia Vinetiana era chiamato a prendere decisioni circa l'utilizzo ed eventualmente la sistemazione degli edifici pubblici a lui affidati. Lo aveva fatto di sicuro a metà anni Ottanta, quando fu fra i tre Provveditori sopra la fabrica del

attenzione al Broletto, dove si continuò ad amministrare la giustizia civile, mentre il grande salone del Consiglio finì per essere utilizzato come magazzino delle granaglie» (DE LEONARDIS 2012:71).

84 VOLTA 1986:68-72, a cui rimando per la biografia di questo ingegnere, utilizzato in quegli anni nei più importanti cantieri della Repubblica, da quello del Ponte di Rialto a quello di Palmanova; altri cenni biografici sono reperibili in MARCHESI 1984:68. Il contenuto del capitolato del 6 marzo 1592 è riassunto in VOLTA 1986:72. Che il Bolda avesse avuto modo di collaborare con Paruta è testimoniato da un dispaccio dei Rettori di Brescia datato 27 luglio 1591 (conservato in: Senato Terra, filza 122): «mandiamo un conto distinto et particolare cavato dalli Zornali tenuti per l'Ingegnero de gli Orzinovi delle spese fatte nel Palazzo della Ressidenza de' Clarissimi Proveditori di quella Terra». Il foglio dei conti allegato è per l'appunto firmato da «Dionisio Boldi Ingegnero». Da una allegata scrittura dei Provveditori sopra le Fortezze si comprende come si tratti della richiesta affinché agli agenti di Orzinovi «siano bonificati li due terzi della spesa fatta per ordine del già Eccellentissimo Proveditor General in Terra Ferma Grimani nel racconciare ed accomodare la Rocca de gl'Orzi Novi, che hora è habitatione delli Proveditori di quella terra». Sull'importanza degli ingegneri (non solo degli architetti) nella cantieristica di stato della Serenissima vd. VALSERIATI 2016:93.

85 I contratti fra il Bolda e i vari imprenditori edili verranno infatti stesi nel corso del 1593: vd. VOLTA 1986:72. In particolare, il Bolda modificò la facciata rivolta ad est del corpo occidentale del Broletto dividendo orizzontalmente i portici, in maniera da creare due piani interni e ricavarne nuovi locali; creò inoltre la balconata ed «un portale dalle forme barocche»: COCCOLI / SCALA / TRECCANI 2009:123-124. 
Palazzo ducale (chiamati cioè a curare il rifacimento interno dell'edificio, dopo i recenti incendi del 1574 e del 1577) $;{ }^{86}$ lo farà nella successiva ambasciata romana, durante la quale si dovrà prendere cura del Palazzo di San Marco, cioè dell'attuale Palazzo Venezia. ${ }^{87}$ In entrambi i casi, vediamo un Paruta che deve, da solo o assieme ai suoi colleghi, prendere delle decisioni non tanto sulla costruzione ex-novo di edifici, quanto su come adattare a nuove esigenze spazi pubblici già pre-esistenti. ${ }^{88}$

86 Si tratta di un incarico minore, spesso ignorato dai biografi dello scrittore veneziano, ma per fortuna non dalla recente voce di Gino Benzoni per il Dizionario Biografico degli Italiani, che almeno riporta la data dell'elezione, ossia il 1583 (BENZONI 2014). L'incarico parutiano non dovette terminare subito, dal momento che nel 1586 lo troviamo a firmare, assieme agli altri due Signori Proveditori sopra la Corografia Francesco Foscari e Giovanni Moro la commissione definitiva al cartografo Cristoforo Sorte (su cui vd. ScHULZ 1990:65-96). Il documento firmato dai tre è riportato per intero in ZANOTTO 1847:126-127 (l'editore ottocentesco però non si è accorto dell'anno more veneto: il "gennaio 1585 " va quindi inteso come "gennaio 1586", come correttamente fa Polinelli 2013/2014:111). Da ricordare, in questa sede, come l'incarico al Sorte comprendesse anche la realizzazione di enormi cartine delle città di Terraferma e del loro contado: fra di esse, anche quella (conservatasi fortunosamente fino ai giorni nostri) del Bresciano: ZaNOTTO 1847:127, Schulz 1990:69-70, LA GRECA 2009:11-12, Polinelli 2013/2014:111. Da notare che nel 1582 (cioè l'anno prima di Paruta) era stato eletto come Provveditore sopra la Corografia Jacopo Contarini (LA GRECA 2009:11, Polinelli 2013/2014:111): si tratta di uno dei personaggi della Perfettione della Vita Politica parutiana, ripubblicata proprio quell'anno in seconda edizione, dopo l'editio princeps del 1579.

87 Palazzo Venezia (affacciato sull'attuale Piazza Venezia, a Roma), dal 1440 abitazione del pontefice di origine veneziana Paolo II (Pietro Barbo) e per questo poi residenza papale, fu donato parzialmente alla Serenissima nel 1564 da Pio IV, così che ivi venissero ospitati gli ambasciatori veneti. Negli ultimi decenni la coabitazione fra ecclesiastici (oltre che residenza papale, una parte del palazzo rimaneva teoricamente patrimonio dei cardinali di S. Marco) si fece problematica, fino a che, nel 1597, Clemente VIII cedette definitivamente il palazzo agli ambasciatori veneziani. Per la storia del Palazzo, vd. BonacCorso 1998:193-198, BARBERINI/DE ANGELIS D’OsSAT/SCHIAVON 2011.

88 Sulla sfida che dovettero fronteggiare i tre Proveditori sopra la Corografia vd. LA GRECA 2009:10. Oltre all'uso di spazi pubblici, l'ambasceria romana fece maturare nel Paruta anche una certa esperienza circa l'affitto degli stessi, come si evince da una lettera diretta a Francesco Barbaro, scritta da Venezia in data 22/08/1598 ed oggi conservata presso: UDINE, Archivio della Curia Arcivescovile Udinese, Epistolario Francesco Barbaro, 905. «Conforme al desiderio et comandamento» del Barbaro (allora Patriarca d'Aquileia, in quel momento a Ferrara), il Paruta invia «la commissione all'Tllustrissimo Signor Ambasciator Mocenico di poter affittar la casa di Roma di Santo 
Per dare un'idea al lettore, si prendano alcuni dispacci della legazione romana, in cui Paruta stesso racconta lo stato pietoso in cui trovò l'ambasciata veneta, e le decisioni che prese a proposito. Giunto nella sua nuova residenza ufficiale, ${ }^{89}$ Paruta afferma che la maggior parte delle stanze ha bisogno di alcun conciero, soprattutto per riparar al coperto, dal momento che piove in moltissimi luoghi, cosa scomoda non solo per il presente ma anche per l'avvenire, «marzendosi le travadure come manifestamente si vede». Dovendo pure pensare al rifacimento di vari porti et balconi rotti,

la spesa non si può sapere precisa essendo il coperto grandissimo et non vedendosi il danno che possa andarsi scoprendo, massime nel ligname fatto per le continue pioggie; pur se mi saranno ballottati scudi ducento procurerò di andarli spendendo con ogni maggior cura et diligenza et così riverentemente ne la prego ${ }^{90}$

Come detto, all'analisi seguirono le decisioni: in una missiva di fine luglio $1594,{ }^{91}$ ad esempio, l'ambasciatore motiva la sua decisione di «far ruinare un pezzo di corridore» (per questo poi da restaurare) per evitare che l'incendio scoppiato nelle adiacenti stalle dell'abitazione del cardinale Valier si diffondesse all'intero Palazzo Venezia. Paruta stava

Spirito a chi si sia in generale, ma con particular nominatione della persona di Monsignor» (poco leggibile, ma pare essere «Matheucci»); eventualmente, il Mocenigo potrà, «se così presterà l'occasione», anche vendere direttamente la casa ubicata in Santo Spirito (probabilmente Santo Spirito in Sassia, vicino al Vaticano). Dopo aver dichiarato il prezzo d'affitto e aver descritto la gestione finanziaria del suo successore (Giovanni Dolfin), Paruta segnala che «in questo affitto è inclusa una casetta, che si dice esser vicina et congiunta con detta casa», facendo presente altresì che «non si trovava il tempo del fine di quella presente locazione». Il monsignore citato dovrebbe essere Girolamo Matteucci (per la cui biografia vd. BRUNELLI 2008): in effetti, in un dispaccio da Roma datato 22/05/1593 (Dispacci, I, pp. 209-210) Paruta scrive che «è giunto in questa città mons. Matteucci, il quale ritorna dal carico di commissario dell'esercito ecclesiastico in Francia».

89 In un estratto della Relazione di Giovan Francesco Marchesini (riportato in BARbERINI/De ANGELIS D'OSsat/SCHIAVON 2011:147), datata 30 maggio 1592, si parla dell'imminente arrivo a Roma del Paruta e della sua incipiente sistemazione a Palazzo Venezia.

90 Dispaccio del 07/11/1592, pubblicato in BARBERINI/DE ANGELIS D’OSSAT/SCHIAVON 2011:148.

91 Dispaccio del 30/07/1594, pubblicato in Dispacci, II, p. 388. 
toccando uno dei problemi nodali dell'edificio ossia la forzata coabitazione del legato laico della Repubblica col Cardinale di San Marco, la quale generava sistematicamente l'insabbiamento dei necessari lavori di restauro, ${ }^{92}$ persino quando (come nel caso Paruta-Valier) i due fossero in ottimi rapporti personali. Se a ciò aggiungiamo le richieste di esborso da parte pontificia, ${ }^{93}$ capiamo bene come l'ambasciatore si trovasse a gestire una situazione ormai fuori controllo. ${ }^{94}$

Tornando al biennio 1591/1592, è forse possibile ricollegare ad un uso pubblico all'interno del Broletto le informazioni che Paruta durante il biennio bresciano chiede, nel suo carteggio col nobiluomo veronese Antonio Serego, ${ }^{95}$ circa i tessuti «per far certo fornimento di

92 «anche a causa della difficile convivenza, [i restauri] furono sempre limitati al minimo indispensabile, con $\mathrm{i}$ risultati che si possono facilmente immaginare» (CASANOVA 1992:228).

93 Dispaccio del 14/01/1595, pubblicato in Dispacci, t. III, p. 16: Clemente VIII chiede che anche i padroni di Palazzo Venezia (come tutti coloro che a Roma possedevano uno stabile) si accollino i lavori di rifacimento della strada pubblica contigua all'edificio.

94 La situazione peggiorò sensibilmente nel corso di qualche anno, come è possibile apprezzare grazie a due dispacci del dicembre 1598 da Roma. Nel primo, datato 05/12/1598, il segretario Giacomo Vendramin fa sapere al Senato che «l'illustrissimo Signor Ambasciator Mocenigo mi fa scriver che dispera di esser qua fra pochi giorni, et io prego il Signor Dio, che lo conduca quanto prima a salvamento, così per suo interesse, come per l'interesse di questo Palazzo della Serenità Sua, qual è in malissimo stato, et con pericolo evidente, quando non sia reparato, di ruinar in molte parti, poiché così nelle sale et camere di sopra, come nelli corritori, quando piove, par a punto esser al scoperto, oltre che ha bisogno anco di molti acconciamenti, et di vetriate, et di impennate» (Roma, c. 276r). Il 26/12/1598 è lo stesso nuovo ambasciatore, Giovanni Mocenigo, a scrivere così: «Io ho trovato, Serenissimo Principe, questo Palazzo in stato molto cativo, poiché non solamente sono cascati alcuni solari della torre per la loro vecchiezza ma in modo rotti li capi, et inmarzito il legno, che fa solaro alle mie stanze et alle sale, che non solamente quando piove restano tutte le stanze piene di acqua guastando tutti li fornimenti di esse, ma se non vi si rimedia presto cascherano ancora, con pericolo de chi vi abita. Ho ritrovato quasi tutti li ... rotti, et a ... quasi tutte le serature, porte et cadenazi delle stanze; onde ha bisogno di presto ... non tanto per sicurezza quanto per honorevolezza publico; però prego la Serenità Vostra a voler venire in qualche risolutione delli suoi ... acciò ch'io possi habitar questo Palazzo, essendovi anco rincresciuto che sia entrata tanta acqua nelli luochi soteranei, che non potrò farla cavare senza grossa spesa» (Roma, cc. 300r-301r).

$95 \mathrm{Nel}$ caso che ci interessa, si sono conservate 10 lettere parutiane scritte da Brescia ad Antonio Serego, consultabili in vd. BIADEGo 1885:12-23. Questa decina di 
muro» e una dozzina di cadrieghe: che il capitano di Brescia, che aveva i suoi appartamenti personali dentro il Broletto, li volesse usare per qualche spazio di ricevimento? ${ }^{96}$

Insomma, mano a mano che si scava nella sua concreta biografia di governo, si mostra sempre di più poco credibile l'immagine tradizionale di un Paruta assimilabile alla schiera di scrittori della Ragion di Stato, costretti a discettare passivamente di una politica diventata ormai opzione del tutto letteraria. L'autore della Perfettione della Vita Politica (1579) e dei postumi Discorsi Politici (1599), al contrario, era chiamato a gestire vari aspetti del ben concreto stato della Repubblica: le sue fortezze e il suo apparato difensivo, le sue attività artigianali (come quella delle armi), il suo approvvigionamento granario, fino ad arrivare ai suoi edifici pubblici.

\section{L'iscrizione AL BROLETTO}

Nonostante quanto esposto fino ad ora, nel 1692 anche la memoria di Paolo Paruta finì nel calderone della furia livellatrice della provisione del Senato, «di cui fecero le spese non solo la vanità barocca di vari rettori ma anche l'onesto scrupolo documentario di tanti loro predecessori».97 Eppure, un insperato aiuto nella ricostruzione storica delle lapidi del Broletto giunge addirittura da Oltremanica, grazie alla preziosa opera di un uomo che in vita venne criticato proprio per quel suo trascrivere lapidi ed epitaffi che ai contemporanei doveva parere opera particolarmente vana: perché - si chiedevano - perdere tempo a ricopiare su carta quello che la pietra conserverà per sempre? ${ }^{98}$

Nel 1611 vennero pubblicate a Londra le Coryat's Crudities:

lettere fornisce ulteriori informazioni sull'incarico bresciano, ad es. circa la reperibilità in città in quegli anni di merci quali olio, frutta, tessuti, cavalli e carrozze, o circa una questione fiscale che vedeva contrapposti i Serego ai Conti di Megli. Sul carteggio fra Paruta e i Serego e sulla sua edizione Biadego (non esente da imprecisioni) vd. le precisazioni filologiche presenti in GIANI 2012:81-83.

96 Per il concetto di comodità nella Brescia dell'epoca vd. BETTONI 2016:76-77.

97 RizZI 1994-1995:629.

98 È Thomas Coryate stesso a raccontare di essersi dovuto difendere dall'accusa, mossagli alcuni Gentlemen inglesi, di essere un tombe-stone traveller perché «I copied out more inscriptions and epitahes [...] that are written upon solid peeces of stone, then any judicious traveller would have done in many yeares» (cit. in PARR 1992:589). 
Hastily gobled up in Five Moneth's Travels, ossia le memorie di viaggio del letterato-avventuriere Thomas Coryate. L'autore, un personaggio alquanto eccentrico, membro della corte del Principe di Galles Enrico Federico Stuart (figlio di Giacomo I), ${ }^{99}$ raccontava il proprio viaggio, compiuto in Italia (per la maggior parte a piedi), ${ }^{100}$ nel corso del $1608,{ }^{101}$ avente come meta Venezia. Il volume ebbe un certo successo all'epoca, come testimoniato per altro dalla sua eredità linguistica. Questo «bold carpenter of words» (come lo ebbe a definire il suo amico Ben Jonson), ${ }^{102}$ infatti, tornò dal Belpaese non solo con delle usanze che dovevano risultare strambe per i suoi conterranei (come l'uso della forchetta, per cui venne soprannominato furcifer negli ambienti di corte), ma anche con delle nuove parole: fu lui, ad esempio, a coniare il sostantivo umbrella, dopo aver osservato il modo in cui gli Italiani tentavano di difendersi dal sole estivo. ${ }^{103}$

Le Crudezze (questa la traduzione italiana del titolo) ${ }^{104}$ di Coryate sono un vero e proprio manuale di viaggio, dotato di informazioni pratiche per il viaggiatore, come prezzi, tassi di cambio, usanze locali, indicazioni sui cibi e le bevande. Naturalmente, non potevano mancare numerosissime descrizioni architettoniche degli edifici visitati, ${ }^{105}$ fra cui quella del Broletto di Brescia, visitato il 15 agosto, ${ }^{106}$ durante il viaggio di ritorno: ${ }^{107}$

99 Per la biografia di Coryate vd. STRACHAn 2003; in lingua italiana, la più recente biografia disponibile è quella contenuta in GıosuÈ 2016:227-232. Il libro doveva già quasi pronto nel novembre del 1610, come dichiarato dallo stesso autore in una lettera, cit. in CRAIK 2004:77.

100 Come riconosciuto scherzosamente dall'immagine (le scarpe di Coryate coronate d'alloro) allegata al contributo encomiastico dello scrittore inglese Henry Peacham, su cui vd. IANNACCARO 2015:51. Sullo status di Coryate come «viaggiatore a basso costo, o viaggiatore fai da te», vd. GIOsuÈ 2016:229.

101 Il viaggio di Coryat durò dal 14 maggio al 3 ottobre 1608 (vd. MARENCO/MEO 1975:41, STRACHAN 2003:285).

102 STRACHAN 2003:286.

103 STRACHAN 2003:286.

104 Per l'esegesi del significato inglese, legato al lessico medico-erboristico, vd. CRAIK 2004:78, IANNACCARO 2015:44.

105 Strachan 2003:286, CRAIK 2004:77.

106 La data è riportata sull'utile mappa presente in PARR 1992:587.

107 IANNACCARO 2015:45. Brescia è teatro di un episodio importante per capire il disgusto che Coryate provava da anglicano per quelle devozioni cattoliche che a lui apparivano idolatriche: «Nel corso di una cerimonia, se pur consapevole delle orribili punizioni che avrebbe dovuto subire per mano dell'Inquisizione se fosse stato colto in flagrante, 
The Palace wherein the Venetian Praetor and Praefectus doe lie (for here bot haue but one Palace, though in other cities they haue two) is a sumptuous building, and furnished with great store of munition and artillery. At the west gate thereof, which is most commonly guarded with a guard of Souldiers that doe attend there all the day with Partizans in their handes, I read this inscription ouer a Scutchin on the right hand as I went into the court.

Dux, Heros, Scriptor Paruta,

Regis, geris, edis,

Vrbem, res, libros,

Imperio, arte, manu.

And this a little vnder the same,

Fide, Virtute, Integritate, Spectatissimo viro

Paulo Parutae buius urbis Praefecto

Optime merito, anno Domini M.D.LXXXXI. ${ }^{108}$

Nel Palace di cui parla Coryat (identificato dai traduttori italiani Marenco e Meo col Broletto) ${ }^{109}$ risiedevano sia il Praetor 'podestà' sia il Praefectus 'capitano' (non particolarmente significativo, in questo caso, l'uso dei termini latini): ${ }^{110}$ si tratta effettivamente della situazione di inizio Seicento, dopo i cambiamenti degli anni Novanta in cui - come abbiamo visto - anche Paruta aveva avuto un piccolo ruolo.

Nessuno stupore anche di fronte al fatto che Coryate trovasse $\mathrm{i}$ soldati ${ }^{111}$ all'ingresso dell'ala occidentale. Nel terzo volume de Il volto storico di Brescia è riportato il particolare di una mappa (datata 1618) de «l'androne di ingresso occidentale al cortile del Broletto e i locali del pianterreno che lo fiancheggiano, destinati quello a sud a

non poté fare a meno di entrare in azione e, contro ogni regola di rispetto e di buona condotta, si spense al punto di rubare un ex-voto in una chiesa» (GIOsuÈ 2016:235).

108 Trascrizione compiuta sull'esemplare Henry E. Huntington Library and Art Gallery, consultato nella copia elettronica presente nel database on-line EEBO (https://eebo.chadwyck.com/, ultimo accesso 23/09/2017). L'iscrizione parutiana è riportata a p. 351.

109 MARENCO/MeO 1975:422.

110 VARANINI (2013:118) ha parlato di un «travestimento nella terminologia della repubblica romana della cariche rettorili nelle città di Terraferma (praetor per podestà, praefectus per capitano, quaestor per camerlengo [...])».

111 A Brescia il viaggiatore inglese non ebbe, a quanto pare, disavventure coi soldati locali, a differenza di quanto gli accadde con una guardia spagnola del Castello Sforzesco di Milano, che lo aggredì verbalmente credendolo fiammingo. Sul curioso episodio vd. IANNACCARO 2015:50-51. 
"Alloggiamento per li soldati", quelli a nord come "Camera fiscale" e "Camerino del denaro" ». ${ }^{12}$ entrando nel cortile del Broletto da quella porta e svoltando a destra (verso sud) si incontravano quindi per forza i souldiers citati da Coryat: non solo perché - come detto da lui - vi stazionavano come guardie all'ingresso, ma anche perché soggiornavano proprio in quella parte del palazzo. ${ }^{13}$

Lo scutchin descritto da Coryate è traducibile in italiano come 'stemma'. ${ }^{14}$ Marenco e Meo traducono la riga di passaggio dalla prima parte («and this a little under the same») come «e un po' sotto la prima, questa», dando forse l'impressione, al lettore italiano, di due diverse lapidi: ma credo che Coryate intenda semplicemente dire che le prime 4 righe erano distanziate dalle ultime 3 (forse perché intervallate da un elemento grafico non descritto, come ad esempio un'arme familiare). ${ }^{115}$ Ci troviamo insomma di fronte ad un unico testo, non a due.

La datazione è denunciata dal testo stesso della lapide: si tratta del 1591, l'unico anno che Paruta spese per intero in città.

Riguardo al testo stesso, è evidente la struttura tripartita, che loda tre diversi aspetti del Paruta: il suo incarico politico di comandante, rettore della città; la sua statura morale di virtuoso individuo (riflessa poi nella sua attività letteraria, visto il suo dialogo sulla Perfettione della Vita Politica, del 1579); il suo incarico di Storiografo Pubblico.

È possibile, oggi, identificare l'iscrizione descritta da Coryate?

Partiamo dalle indicazioni spaziali presenti nel testo: il punto di vista dell'osservatore deve essere interno al cortile, non solo perché prima di descrivere l'iscrizione dichiara di entrare «into the court» ma

112 AAVV 1980:164. Si tratta di una mappa oggi conservata in Archivio di Stato a Venezia, disegnata da Giovanni Battista Lantana (1581-1627) e allegata ad un dispaccio del 1618 di Antonio Mocenigo.

113 Già nel 1530 le autorità veneziane avevano deciso di far spostare in Broletto la gendarmeria, che prima era situata nell'attuale Piazza della Loggia: vd. VOLTA 1987:22. Le armi che i soldati tengono in mano sono delle Partizans, tradotte da Marenco e Meo come 'partigiane' (MARENCO/MEO 1975:351); nella prima edizione (1598) del suo dizionario, Florio propone partesan come prima possibile traduzione (l'altra è iavelin) dell'it. partesana: vd. FLORIO 1972:259.

114 MARENCO/MEO 1975:351. Florio propone scutchion come quarta possibile traduzione dell'it. scudo: FLORIO 1972:359.

115 Come esempio di questo utilizzo di «..a little...» nelle descrizioni iconografiche di Coryate, vd. la descrizione di un dipinto di Palazzo Ducale cit. in Wolters 1987:253. 
anche perché dopo averlo fatto (in un pezzo non riportato in questa sede) Coryate afferma che «tutte le pareti incontro [nel cortile del palazzo] sono ornate da diversi stemmi di gentiluomini veneziani». ${ }^{116}$ Per questi due motivi possiamo senza problemi escludere sia la lapide a forma di cartiglio della porta occidentale esterna (su Piazza Paolo VI), i cui resti attuali rimandano peraltro senz'ombra di dubbio alla posteriore Guerra di Candia, ${ }^{117}$ sia il leone di San Marco abraso ${ }^{118}$ sottostante il «bassorilievo quattrocentesco in pietra raffigurante la Giustizia» ${ }^{119}$ (entrambi nell'androne di ingresso).

Concentrando quindi la nostra attenzione sulla parete interna (orientata ad est) dell'ala occidentale, ${ }^{120}$ oggi ${ }^{121}$ possiamo osservare ancora sei lapidi superstiti, ${ }^{122}$ tutte concentrate attorno alla porta d'ingresso, e (purtroppo) perfettamente abrase. ${ }^{123}$ Poco si può dire di più, ma forse

116 MARENCO/MeO 1975:351.

117 «Alla fine del ‘600, proprio quando si eradono tutte le lapidi del palazzo per una dura disposizione veneziana (3 marzo 1692), il provveditore Pietro Morosini vuole ricordare il glorioso congiunto Francesco (distruttore, ahimè, del Partenone nel 1687) con la seguente iscrizione sugli abaci della bella porta occidentale (che dà sulla piazza del Duomo) "Naupacto, Neocastro expugnatis, Peloponneso subacta, finibus Dalmatiae ampliatis"» (PIOVANELLI 1975:30).

118 In RIZZI 1994-1995:644 si trova il catalogo dei quattro leoni marciani scolpiti perduti del Broletto dopo le devastazioni giacobine del 1797, a causa delle quali non rimane che affermare che «di leoni marciani Brescia è avarissima» (RIZZI 19941995:627).

119 ZANE 1998:7.

120 Per il prospetto stratigrafico del Prospetto 2 (cioè il fronte interno est del corpo ovest) vd. la Tavola 5 di CocCOli / SCALA / TreCCAni 2009:127. Per le foto del prospetto in questione, vd. PIOVANELli 1975:19, VOLTA 1987 e MARCONI 1993.

121 Sopralluogo compiuto il 18 luglio 2017 assieme al dott. Luca Tizzano, che ringrazio in questa sede per avermi accompagnato.

122 Ossia, seguendo la numerazione presente in CocCOli / SCAlA / TRECCANI 2009:127, gli items 1035, 1043, 1091, 1093, 1095, 1100. Non è nemmeno da escludere che, col passare dei secoli, la lapide sia stata spostata su un altro lato del cortile (quello orientale, ad es., che ne presenta moltissime, una vera e propria «teoria di lapidi e bassorilievi completamente abrasi ed illeggibili»: ZANE 1998:8), o in un altro luogo all'interno del Broletto. Segnalo ad es. come nella Fig. 3 di PANAZZA 1964/1965, ossia il prospetto di questo lato del Broletto nel 1914, prima dei restauri di Giovanni Tagliaferri, si intraveda (purtroppo la qualità dell'immagine non permette di giudicare meglio) sotto l'attuale balcone quella che potrebbe essere una lapide rettangolare, posizionata ancora più a sinistra e ancora più in basso rispetto a quella che attualmente si trova più a sinistra.

123 «Non solo furono rimossi busti e cancellate iscrizioni ma persino i comparti 
la maggiore indiziata rimane quella più a sinistra, ${ }^{124}$ la quale si differenzia dalle altre per una piccola decorazione su ciascuno dei quattro lati: entrando nel cortile secondo le indicazioni di Coryate, infatti, è l'unica che si può trovare on the right hand, essendo tutte le altre centrali rispetto alla porta.

In conclusione, rimane aperta un'ultima domanda: perché Coryate si prese la briga di trascrivere il testo proprio di tale lapide? Una casuale curiosità intercettata dal suo sguardo onnivoro, ${ }^{125}$ affammato soprattuto di epigrafi, emblemi e epitaffi? ${ }^{126} \mathrm{Il}$ segno di una stima per il Paruta autore ${ }^{127}$ da parte del letterato inglese, ${ }^{128}$ che - come ha dimo-

araldici furono scalpellati trasformandoli in informi lastre. In tale contesto ai Giacobini di un secolo dopo non restava molto da fare se non eliminare i leoni marciani che ovviamente erano stati fino ad allora risparmiati» (RizZI 1994-1995:629).

124 Secondo la numerazione prima citata, la n. 1091.

125 Nei versi latini di prefazione alle sue Crudities, Coryate «announces that the text is half-baked (cruda), but nevertheless invites the reader to digest it» (CRAIK 2004:81).

126 Coryate infatti «fu quello che gli Inglesi chiamerebbero un crank, ma non privo tuttavia di cultura e di acume: era infatti un discreto grecista e latinista, che colleziona e discute con passione tutte le epigrafi, gli emblemi, gli epitaffi in cui s'imbatte» (REBORA 1952:142-143); proprio a causa di ciò si è voluto riconoscere nel viaggiatore inglese i segni di una genuina passione antiquaria (REBORA 1952:145). Sulle epighraphical transcriptions delle Crudities vd. anche PALMER 2014:339, GiosuÈ 2016:233.

127 Il fatto aprirebbe scenari molto interessanti, perché la prima traduzione in inglese di un'opera parutiana risale al 1627 (sulla ricezione di Paruta nel Regno Unito vd. CERVELLI 1967 e GIANI 2012:36). Tuttavia, che le opere parutiane fossero già disponibili negli ambienti di corte frequentati da Coryate è dimostrato dal fatto che il grande lessicografico italo-inglese John Florio, come segnalato in MARAZZINI 2000:702, cita Paolo Paruta come fonte. Non c'è da stupirsi: l'autore del grande dizionario italianoinglese, infatti, «cita nella prefazione settantadue fonti scritte diverse per la prima edizione e ben duecentocinquantadue per la seconda (come termine di confronto, la prima edizione del Vocabolario degli Accademici della Crusca, pubblicata nel 1612, elenca duecentotrenta opere come fonti, ventidue meno di Florio)»(FERGUSON 2012:68). Le due edizioni di Florio appena citate (su cui vd. FERGUSON 2012:57) sono rispettivamente la prima, ossia A Worlde of Wordes (1598), e la seconda, ampliata, cioè Queen Anna's New World of Words (1611). Sulla presenza di Machiavelli, Botero e Paruta nella seconda di queste opere vd. anche PIRILLO 2013:136.

128 Katharine Craik ha posto l'accento sull'ambiguità di Coryate circa il tema della politica: l'autore delle Crudities in certi punti del diario di viaggio cita loda le opere di ambasciatori e statisti (fra cui Gasparo Contarini), in certi altri dichiara la sua estraneità al tema (ad. es. «because I am a private man and no statist, matters of policie are impertinent unto me»): vd. CRAIK 2004:85. Piero Rebora pare propendere per la 
strato Rita Severi ${ }^{129}$ - spesso sceglieva le città in cui fare sosta in base agli autori da lui amati? Il frutto dei racconti dei locali che, ancora dieci anni dopo la morte del capitano e quasi vent'anni dopo l'incarico, ne conservavano una buona memoria, tanto da additare all'Inglese di passaggio in città la lapide posta anni addietro in onore del capitano veneziano? Al momento, non è possibile dare una risposta certa a questa domanda: ma certo ulteriori studi sulle fonti potranno aiutarci a fare luce non solo su questa, ma anche su tutte quante le altre lapidi abrase del Broletto bresciano.

\section{BIBLIOGRAFIA}

\section{Opere di Paolo Paruta}

Tutte le opere vengono citate con il rispettivo numero di paragrafo (preceduto dall'eventuale numero di libro), seguendo - ove possibile - il testo delle trascrizioni elettroniche liberamente consultabili sul database on-line Parutiana, accessibile all'indirizzo https://unive.academia.edu/MarcoGiani/Parutiana (ultimo accesso: 09/09/2017). Si rimanda alla stessa fonte per le informazioni sulle singole edizioni di riferimento.

Dispacci

R. Fulin, F. Stefani (a cura di): La legazione di Paolo Paruta, Monumenti editi della R. Deputazione Veneta di Storia Patria, serie IV (3 voll.), Venezia, Visentini, 1887-1888

DP Discorsi Politici

NuovaInfo Giovanni Paruta, Nuova Informazione

II. Fonti archivistiche Capi di $X$

VenezIA, Archivio di Stato, Capi del Consiglio di dieci, Dispacci (lettere) dei rettori e pubblici rappresentanti, 25.

seconda interpretazione: «Contrariamente agli altri viaggiatori inglesi che lo precedettero, Coryat non mostra molto interesse per l'aspetto politico e religioso della vita italiana» (REBORA 1952:144, ma vd. anche GIOSUÈ 2016:229); Giuliana Iannaccaro ha sottolineato come «le questioni politiche non solo lo riguardano, ma sono anche materia pericolosa, dalla quale i viaggiatori farebbero bene a tenersi lontani per non incorrere in incidenti che possono rivelarsi anche tragici» (IANNACCARO 2015:47). Sul tentativo di Coryate di «turn travel into a literary event, to rescue it from the new mediocrity of tourism» vd. PARR 1992:586.

129 SEVERI 2009:139-143. Come esempio emblematico viene portato quello di Mirandola, patria di Pico, che tuttavia lasciò deluso il viaggiatore inglese, il quale «non potè che constatare che Mirandola invece d'essere la città ideale del mito platonico, è un covo di briganti». 
Roma

Senato Terra

III. Studi e testi

AAVV 1978

AAVV 1980

Balistreri 2015

Barbiroli 2012

Bauce 2010

Benucci 2001

Benucci 2007

Benzoni 2014

Bettoni 2016

Biadego 1885

Bonaccorso 1998

Bonelli 1924

Bowd 2010

Brunelli 2008
Venezia, Archivio di Stato, Senato, Dispacci, Dispacci degli ambasciatori e residenti, Roma, filza 42.

Venezia, Archivio di Stato, Senato, Deliberazioni, Terra.

Il volto storico di Brescia - Volume primo, Brescia, Grafo edizioni, 1978.

Il volto storico di Brescia - Volume terzo, Brescia, Grafo edizioni, 1980.

E. Balistreri, Le Istituzioni politiche, giudiziarie ed amministrative di Venezia. In: idem, Prontuario delle Istituzioni e delle Magistrature di Venezia, Roma, Aracne, pp. 45-90.

Barberini/De Angelis d'Ossat/Schiavon 2011 M. G. Barberini, M. De Angelis d'Ossat / A. Schiavon, La storia del Palazzo di Venezia : dalle collezioni Barbo e Grimani a sede dell'Ambasciata veneta e austriaca, Roma, Gangemi, 2011.

B. Barbiroli, Repertorio storico degli archibugiari italiani dal XIV al XX secolo, Bologna, CLUEB, 2012.

F. Bauce, La politica economica bresciana tra Venezia, Milano e l'Europa nei secoli XV-XVI, Studi Storici Luigi Simeoni 60 (2010), 31-45.

F. Benucci, Le università dello Studio di Padova per i rettori della città, Quaderni per la storia dell'Università di Padova, 34 (2001), 243-279.

F. Benucci, Stemmi di scolari dello Studio patavino fuori delle sedi universitarie, Treviso, Antilia, 2007.

G. Benzoni, Paruta, Paolo, in Dizionario Biografico degli Italiani 81, Roma, Istituto della Enciclopedia Italiana, 482-487.

B. Bettoni, Stili di vita e interni domestici del patriziato bresciano nel secondo Cinquecento, in F. Piazza / E. Valseriati (a cura di), Brescia nel secondo Cinquecento: architettura, arte e società, Brescia, Morcelliana, 2016, 75-91.

G. Biadego, Lettere inedite di Paolo Paruta, Verona, Goldschagg, 1885.

G. Bonaccorso, I Veneziani a Roma da Paolo II alla caduta della Serenissima: l'ambasciata, le fabbriche, il quartiere, in D. Calabi, P. Lanaro (a cura di), La città italiana e $i$ luoghi degli stranieri, XIV-XVIII secolo, Bari/Roma, Laterza, 1998, 192-205.

G. Bonelli, L'archivio di Stato in Brescia - Notizia e inventario. Pavia, Scuola Tipografica Artigianelli, 1924.

S. Bowd, Venice's Most Loyal City. Civic Identity in Renaissance Brescia, Cambridge/London, Harvard University Press, 2010.

G. Brunelli, Matteucci, Girolamo, in Dizionario Biografico degli Italiani 72, Roma, Istituto della Enciclopedia Italiana, 272-274 
Casanova 1992 M. L. Casanova, Palazzo Venezia, Roma, Editalia, 1992.

Casella 2009

L. Casella, Savorgnan, Giulio, In Nuovo Liruti: Dizionario Biografico dei Friulani. 2. L'Età Veneta, 2009, 2266-2273.

Cervelli 1967

I. Cervelli, Giudizi seicenteschi dell'opera di Paolo Paruta, Annali dell'Istituto italiano per gli studi storici 1 (1967-68), 237-308.

Coccoli/Scala/Treccani 2009 C. Coccoli / B. Scala / G. P. Treccani, Stratigrafie e restauri al Broletto di Brescia, Archeologia dell'Architettura 14 (2009), 105-138.

Codagli 1592 D. Codagli, L'Historia Orceana [...] del R. P. F. Domenico Codagli, predicatore. Nella quale si trattano le guerre et le cose aunenute in questa sua patria, ch'abbracciano quasi dua milla anni, Brescia, Gio. Battista Borella, 1592.

Corazzol 1997 G. Corazzoli, Cineografo di banditi su sfondo di monti: Feltre 1634-1642, Milano, UNICOPLI, 1997.

Cozzi 1963-1964 Cultura, politica e religione nella "pubblica storiografia" veneziana del Cinquecento, Bollettino dell'Istituto di Storia della Società e dello Stato V-VI (1963-1964), ora in: idem, Ambiente veneziano, ambiente veneto, Venezia, Fondazione Giorgio Cini/Marsilio, 1997, 13-86.

Craik 2004 K. A. Craik, Reading "Coryats Crudities" (1611), Studies in English Literature 1500-1900 44 (2004), 77-96.

De Leonardis 2012 F. De Leonardis, Signori e cittadini sul palcoscenico del potere, in Il Broletto di Brescia, Brescia, Grafo edizioni, 2012, 59-82.

Favaro 1891 A. Favaro, Lettere passate tra Antonio Riccobono et il Procuratore Paruta d'intorno allo scrivere le Historie venete, Archivio Veneto 2 (1891), 169-180.

Ferguson 2012 R. Ferguson, Primi influssi culturali italo-veneti sull'inglese: la testimonianza dei venezianismi in Florio, Coryate e Jonson, Quaderni Veneti (n. s. digitale) 1 (2012), 57-82.

Florio 1972 J. Florio, A Worlde of Wordes (1598), Hildesheim/New York, Georg Olmes Verlag, 1972.

Gaibi 1964 A. Gaibi, Le armi da fuoco, in AAVV (a curia di), Storia di Brescia. III. La dominazione veneta (1576-1797), Brescia, Morcelliana, 1964, pp. 819-884.

Galtarossa 2013 M. Galtarossa, Le politiche culturali per la Cancelleria ducale, in A. Caracausi / A. Conzato (a cura di), Formazione alla politica, politica della formazione a Venezia in Eta moderna, Roma, Viella, 2013, 73-100.

Gamba 2016 G. Gamba, Il vescovo Domenico Bollani e la città di Brescia, fra fede e rinnovamento. in F. Piazza / E. Valseriati (a cura di), Brescia nel secondo Cinquecento: architettura, arte e società, Brescia, Morcelliana, 2016, 53-73.

Giani 2012 M. Giani, Paolo Paruta: il lessico della politica. Tesi dottorale 
Giani 2013/2014

Giani 2014

Giani 2014b

Giani 2016

Giani 2017

Giosuè 2016

Guerrini 1922

Iannaccaro 2015

La Greca 2009

Lanaro 1979 discussa presso Università $\mathrm{Ca}$ ' Foscari (Venezia), relatore Francesco Bruni, correlatore Jean-Louis Fournel, http://hdl. handle.net/10579/1212 [Ultimo accesso: 23/09/2017]

M. Giani, Grano bavarese a Venezia: Progetti di tratte transalpine in una lettera di Minuccio Minucci a Paolo Paruta (1597), Atti dell'Istituto Veneto di Scienze, Lettere ed Arti 172 (2013/2014), Classe di scienze morali, lettere ed arti, 371-440.

M. Giani, Athenian ostracism in Venetian disguise: an historical diatribe in late Renaissance Italy, P. M. Kitromilides (a cura di) Athenian Legacies. European Debates on Citizenship, Firenze:, Olschki, 2014, 179-193.

M. Giani, Il ruolo e il fine delle membra della repubblica nel pensiero politico di Paolo Paruta, in J.-L. Fournel/ H. Miesse/ P. Moreno/ J.-C. Zancarini (a cura di), Catégories et mots de la politique à la Renaissance italienne / Categorie e termini della politica nel Rinascimento italiano, Bruxelles-Wien, Peter Lang, 2014, pp. 87-102.

M. Giani, Paolo Paruta (1540-1598): Un lessico al crocevia, in R. Librandi / R. Piro (a cura di), L'italiano della politica e la politica per l'italiano (Atti del XI Convegno ASLI Associazione per la Storia della Lingua Italiana, Napoli, 20-22 novembre 2014), Firenze, Franco Cesati, 2016, 191-203.

M. Giani, La Repubblica di Venezia e l'assedio di Malta - Una causa veneziana fra Paolo Paruta e Angelo Dolfin (1565), Studi Veneziani 75 (2017), 223-314.

D. Giosuè, Thomas Coryate: buffone di corte, viaggiatore, fachiro, in G. Platania (a cura di), Pot-pourri. Studi in onore di Silvana Ferreri, Viterbo, Sette Città, 225-248.

Giové Marchioli 2010 N. Giové Marchioli, Cancellare il ricordo: la damnatio memoriae nelle iscrizioni medievali tra formule e scalpellature, in I. Lori Sanfilippo/ A. Rigon (a cura di), Condannare all'oblio: pratiche della damnatio memoriae nel Medioevo, Roma, Istituto Storico Italiano per il Medio Evo, 2010, 127-156.

P. Guerrini, Fonti per la storia bresciana - V, Brescia, Edizioni del Moretto, 1922.

G. Iannaccaro, Le 'Crudezze' di Thomas Coryat (1611), un viaggiatore inglese nella Milano spagnola, in M. V. Calvi / E. Perassi (a cura di): Milano città delle culture, Roma, Edizioni di Storia e Letteratura, 2015, 43-52.

O. La Greca, Carte geografiche riservate. Ricognizione sulle corografie sotto chiave in Palazzo ducale, Geografia, 32 (2009), 9-14. P. Lanaro, Introduzione alle Relazioni dei provveditori di Asola e Orzinuovi e dei podestà e capitani di Crema, in Relazioni dei rettori veneti in Terraferma - XIII. Podestaria e capitanato di Crema 
Lanaro 1999

Laven 1994

Mainoni 1997

Manno 1986

Manno 1987

Marazzini 2000

Marchesi 1984

Marenco/Meo 1975

Marconi 1993

Mazzoldi 1964

Mazzuchelli 1732

Montanari 2007

Morin/Held 1980

Morin 1981

Netto 1991/1992
- Provveditorato di Orzinuovi - Provveditorato di Asola. Milano, Giuffrè, 1979, XV-XLIX.

P. Lanaro, I mercati nella Repubblica Veneta. Economie cittadine e stato territoriale (secoli XV-XVIII), Venezia, Marsilio, 1999.

P. Laven, Banditry and lawlessness on the Venetian Terraferma in the later Cinquecento, in T. Dean/ K. J. P. Lowe (eds.): Crime, society and the law in Renaissance Italy. Cambridge: Cambridge University Press, 1994, 221-248.

P. Mainoni, Le radici della discordia. Ricerche sulla fiscalità a Bergamo tra XIII e XV secolo, Milano, UNICOPLI, 1997.

A. Manno, Politica ed architettura militare: le difese di Venezia (1557-1573), Studi Veneziani 11 (1986), 91-137.

A. Manno, Un compromesso fra "vecchi" e "giovani": il nuovo castello di Brescia, Studi Veneziani 13 (1987), 255-284.

C. Marazzini, The teaching of Italian in 15th-and 16th-century in Europe, in S. Auroux, Sylvain / E. F. K. Koerner / H.-J. Niederehe / K. Versteegh (a cura di): History of the Language Sciences - vol. 1, Berlin/New York, De Gruyter, 2000, 699-705. P. Marchesi, Fortezze veneziane: 1508-1797, Milano, Rusconi, 1984.

F. Marenco / A. Meo (a cura di): Thomas Coryat / Crudezze: viaggio in Francia e in Italia, 1608, Milano, Longanesi,

P. Marconi, Il Broletto di Brescia: filologia e progetto. La riabilitazione di un palinsesto architettonico degradato ma prezioso, Brescia, Grafo edizioni, 1993.

L. Mazzoldi, Gli ultimi secoli del dominio veneto - L'economia nei secoli XVII E XVIII, in Storia di Brescia. III. La dominazione veneta (1576-1797), Brescia, Morcelliana, 3-145.

G. M. Mazzuchelli, Raccolta di privilegj, ducali, giudizj, terminazioni, e decreti pubblici sopra varie materie giurisdizionali, civili, criminali, ed Economiche, concernenti la Città, e Provincia di Brescia, Brescia, Bossino, 1732.

D. Montanari: Brescia religiosa tra Cinquecento e Seicento, in G. Archetti (a cura di), Inquirere Veritatem. Studi in memoria di mons. Antonio Masetti Zannini, I, 323-338.

M. Morin/R. Held, Beretta: la dinastia industriale più antica del mondo, Chiasso, Acquafresca, 1980.

M. Morin, La produzione delle armi da fuoco a Gardone Val Trompia, in Armi e cultura nel Bresciano 1420-1870, Brescia, F.lli Geroldi, 1981, 67-76.

G. Netto, 15 dicembre 1691: siano distrutte tutte le iscrizioni ed altre memorie, laudative dei podestà veneziani, innalzate nelle città suddite, Atti e memorie dell'Ateneo di Treviso 9 (19911992), 13-89. 
Palmer 2014

Panazza 1964/1965

Parr 1992

Pasero 1940

Passamani 1987

Piovanelli 1975

Pirillo 2013

Polinelli 2013/2014

Pompeati 1905

Raines 2006

Rapaggi 2012

Rebora 1952

Rizzi 1994/1995

Sapienza 2007/2008

Schulz 1990
P. S. Palmer, 'The progress of thy glorious book': Material Reading and the Play of Paratext in Coryats Crudities (1611), Renaissance Studies 28.3, 336-355.

G. Panazza, Appunti per la storia dei Palazzi Comunali di Brescia e Pavia, Archivio Storico Lombardo (s. IX) 91/92 (1964/65), 181-203.

A. Parr, Thomas Coryat and the Discovery of Europe, Huntington Library Quarterly 55 (1992), 578-602.

C. Pasero, Documenti e notizie intorno all'industria delle armi a Brescia durante il secolo XVI, in Atti e memorie del Quarto congresso storico lombardo: Pavia, 18-19-20 maggio 1939, Milano, Giuffré, 287-298.

B. Passamani, Brescia e Ceruti: patrizi, popolo, pitocchi. Alla ricerca di "fatti certi" e di "persone vive", in Giacomo Ceruti il Pitocchetto, Milano, Mazzotta, 1987, 11-28.

G. Piovanelli, Segreti e curiosità del Broletto di Brescia e dei palazzi comunali italiani, Bornato, Franco Sardini, 1975.

D. Pirillo, Republicanism and Religious Dissent: Machiavelli and the Italian Protestant Reformers, in A. Arienzo/A. Petrina (eds.) Machiavellian encounters in Tudor and Stuart England: literary and political influences from the Reformation to the Restoration, Farnham, Ashgate, 2013, 121-140.

M. Polinelli, I molti mestieri dell' architetto nel Cinquecento: il caso di Cristoforo Sorte, tesi di laurea specialistica, relatrice Elisabetta Molteni, Università Ca Foscari (Venezia). http://hdl.handle. net/10579/5084 [Ultimo accesso: 20/07/2017]

A. Pompeati, Per la biografia di Paolo Paruta, Giornale Storico della Letteratura Italiana 23 (1905), 48-66.

D. Raines, L'invention du mythe aristocratique - L'image de soi du patriciat vénitien au temps de la Sérénissime, Venezia, Istituto Veneto di Scienze, Lettere ed Arti, 2006.

A. Rapaggi, Le secolari riscritture di uno spazio identitario, in AAVV, Il Broletto di Brescia, Brescia, Grafo edizioni, 5-29.

P. Rebora, Momenti di cultura italiana e inglese: saggi e ricerche, Mazara, Società Editrice Siciliana, 1952.

A. Rizzi, L'emblema della Serenissima nel Bresciano, Atti dell'Istituto Veneto di Scienze, Lettere ed Arti - Classe di scienze morali, lettere ed arti 153 (1994-1995), 627-687.

V. Sapienza, (Intorno a) Leonardo Corona (1552-1596): documenti, fonti e indagini storico-contestuali. Tesi di dottorato, relatori Augusto Gentili e Maurice Brock, Università Ca' Foscari, http://hdl.handle.net/10579/1130 (ultimo accesso: 20/07/2017). J. Schulz, La cartografia tra scienza e arte: carte e cartografi nel Rinascimento italiano, Modena, Panini, 1990. 
Seneca 1959

Severi 2009

Signaroli 2017

Strachan 2003

Tagliaferri 1978

Valseriati 2016

Varanini 2013

Volta 1986

Volta 1987

Wolters 1986

Zane 1998

Zanotto 1847

Zeno 1718
F. Seneca, Il doge Leonardo Donà, la sua vita e la sua preparazione politica prima del dogado, Padova, Antenore, 1959.

R. Severi, Rinascimenti: Shakespeare \& Anglo/Italian relations, Bologna, Patron, 2009.

S. Signaroli, Domenico Molino e Isaac Casaubon, Milano, CUSL, 2017.

M. Strachan, Coryate, Thomas (c. 1577-1617), in J. Speake (ed.), Literature of Travel and Exploration: an Encyclopedia - Volume 1, 285-87.

A. Tagliaferri, Relazioni dei rettori veneti in Terraferma. XI: Podestaria e Capitanato di Brescia, Milano, Giuffré, 1978.

E. Valseriati, I Deputati alle pubbliche fabbriche e gli architetti comunali (1538-1597), in F. Piazza / E. Valseriati (a cura di), Brescia nel secondo Cinquecento: architettura, arte e società, Brescia, Morcelliana, 2016, 93-126.

G. M. Varanini, "Entrar in Consiglio". Esperienze di avvio alla vita politica e amministrativa nella Terraferma quattrocentesca. in A. Caracausi / A. Conzato (a cura di), Formazione alla politica, politica della formazione a Venezia in Eta moderna, Roma, Viella, 2013, 101-123.

V. Volta, Restauri ed architetti del Broletto in Cittadella Nova, AB. Atlante Bresciano 7 (1986), 67-78.

V. Volta, Il palazzo del Broletto di Brescia. Brescia: Banca San Paolo di Brescia.

W. Wolters, Guerra e pace nei dipinti di Palazzo Ducale, in AAVV, Venezia e la difesa del Levante: da Lepanto a Candia 1570-1670, Venezia, Arsenale, 1986, 247-254.

M. Zane, Le otto mura del Broletto, Brescia, AreaMarket, 1998. F, Zanotto, Il Palazzo Ducale di Venezia, Venezia, Antonelli.ì, 1847. A. Zeno, Degl'istorici delle cose veneziane, $i$ quali hanno scritto per pubblico decreto, Venezia, Lovisa, 1718. 